TADEUsz RADZIK (Wrocław)

\title{
POORLY CONVEX FUNCTIONS AND THEIR APPLICATION TO AN OPTIMIZATION PROBLEM
}

Abstract. The paper introduces a new class of functions, called poorly convex, defined on convex subsets of $\mathbb{R}^{n}$. The class is bigger than the class of classical convex functions, and is a subset of the class of quasi-convex ones. The theory of poorly convex functions is developed, and its application to an optimization problem is shown.

1. Introduction. Convex functions play an important role in domains as diverse as optimization, economics and engineering. However, for many problems the classical notion of convexity no longer suffices, and different types of "generalized convexity" like pseudo-convexity, quasi-convexity, etc. are necessary. We mention here the early work by de Finetti (1949), Fenchel (1953), Arrow and Enthoven (1961), Mangasarian (1965), Karamardian (1967), and Hanson (1981). Some further results and a review of the latest developments in this area can be found in Guerraggio and Molho (2004), Islam and Craven (2005), Matkowski (2008) and Mishra, Wang and Lai (2009).

In the recent paper of Radzik (2014), a new class of functions, called poorly convex, considered on intervals of the real line, is introduced, and the theory of such functions is studied. The notion of poor convexity naturally generalizes classical convexity, while being stronger than quasi-convexity.

In this paper we generalize poor convexity from $\mathbb{R}$ to any euclidean space $\mathbb{R}^{n}$, considering poorly convex functions defined on convex subsets of $\mathbb{R}^{n}$. Relying on some results obtained in Radzik (2014), we develop a theory of poorly convex functions on $\mathbb{R}^{n}$, showing many properties of such

2010 Mathematics Subject Classification: 26B25, 46N10, 52A41.

Key words and phrases: poorly convex function, convexity, quasi-convexity, pairwise poorly convex family, optimization problem, Nash equilibrium.

Received 21 March 2019; revised 22 July 2019.

Published online 31 January 2020. 
functions and their families. We also present an application of the results obtained to some general optimization problem.

The organization of the paper is as follows. Section 2 is devoted to basic definitions and some background results. In Section 3, a review of results on poorly convex functions defined on intervals in $\mathbb{R}$ is given. Some of them come from Radzik (2014).

In Section 4, a complete characterization of poorly convex functions and pairwise poorly convex families of functions defined on convex subsets of $\mathbb{R}^{n}$ is given. In Section 5, we present the main theorem of this paper, which describes a surprising property of those families. Four examples of pairwise poorly convex families are given in Section 6. In Section 7, we present an application of the theory of poorly convex functions to an optimization problem.

2. Preliminary notions and definitions. Let $n \geq 1$ be a natural number. Throughout, $\mathbb{U}$ denotes a convex subset of $\mathbb{R}^{n}$. All the functions considered are real-valued.

We will use the classical lexicographic ordering in $\mathbb{R}^{n}$ : for $s=\left(s_{1}, \ldots, s_{n}\right)$, $t=\left(t_{1}, \ldots, t_{n}\right) \in \mathbb{R}^{n}$, we write $s \prec t$ if $s_{i}<t_{i}$ for the smallest index $i$ with $s_{i} \neq t_{i}$. As usual, $s \preceq t$ if $s \prec t$ or $s=t$.

To begin, we recall the classical definitions of quasi-convex and lower semicontinuous functions.

Definition 1. A function $f$ on $\mathbb{U}$ is called quasi-convex if for any $c \in \mathbb{R}$ the set $\{u \in \mathbb{U}: f(u) \leq c\}$ is convex. If this holds for the reverse inequality, $f$ is called quasi-concave.

Definition 2. A function $f$ on $\mathbb{U}$ is called lower semicontinuous if for any $c \in \mathbb{R}$ the set $\{u \in \mathbb{U}: f(u) \leq c\}$ is closed. In case of the reverse inequality, $f$ is called upper semicontinuous.

Now we recall several classical properties of quasi-convex and lower and upper semicontinuous functions.

Proposition 1.

(a) A function $f$ on $\mathbb{U}$ is quasi-convex [lower semicontinuous] if and only if $-f$ is quasi-concave [upper semicontinuous]. If $f$ is linear then it is both quasi-convex and quasi-concave. If $f$ is continuous then it is both lower and upper semicontinuous.

(b) If $f_{1}, \ldots, f_{k}$ are lower [upper] semicontinuous functions on $\mathbb{U}$ and $x_{1}, \ldots$, $x_{k} \geq 0$, then the function $\sum_{i=1}^{k} x_{i} f_{i}$ is also lower [upper] semicontinuous on $\mathbb{U}$. 
(c) A function $f$ is lower [upper] semicontinuous on $\mathbb{U}$ if and only if for each $u_{0} \in \mathbb{U}$

$$
\liminf _{u \rightarrow u_{0}} f(u) \geq f\left(u_{0}\right) \quad\left[\limsup _{u \rightarrow u_{0}} f(u) \leq f\left(u_{0}\right)\right]
$$

(d) Any lower [upper] semicontinuous function $f$ defined on a compact set $\mathbb{U}$ achieves its minimal [maximal] value at some point of $\mathbb{U}$.

(e) For any family $\left\{f_{\alpha}\right\}$ of lower [upper] semicontinuous functions $f_{\alpha}$ on $\mathbb{U}$, the function $F$ defined by $F(u)=\sup _{\alpha} f_{\alpha}(u)\left[F(u)=\inf _{\alpha} f_{\alpha}(u)\right]$ is lower [upper] semicontinuous on $\mathbb{U}$.

The next two definitions are basic for our paper. They describe some generalization of convex and concave functions. Before giving them, we need some additional notation. Set

$$
\begin{aligned}
P_{\mathbb{U}} & :=\left\{\left(u_{1}, u_{2}, \lambda\right) \in \mathbb{U}^{2} \times(0,1): u_{1}, u_{2} \in \mathbb{U}, u_{1} \prec u_{2}, \text { and } 0<\lambda<1\right\}, \\
Q_{\mathbb{U}} & :=\left\{\bar{u} \in \mathbb{U}^{3}: u_{1} \prec u_{3} \text { and } u_{2}=\lambda u_{1}+(1-\lambda) u_{3} \text { for some } 0<\lambda<1\right\},
\end{aligned}
$$

where $\bar{u}:=\left(u_{1}, u_{2}, u_{3}\right)$. Note that

$$
\bar{u} \in Q_{\mathbb{U}} \Longrightarrow u_{1} \prec u_{2} \prec u_{3},
$$

which in case of $\mathbb{U}=[a, b] \subset \mathbb{R}$ can be modified to

$$
\bar{u} \in Q_{[a, b]} \Longleftrightarrow\left[a \leq u_{1}<u_{2}<u_{3} \leq b\right] .
$$

Definition 3. A function $f$ on $\mathbb{U}$ is called poorly convex [poorly concave] if there is a function $p: P_{\mathbb{U}} \rightarrow(0,1)$ such that for all $\left(u_{1}, u_{2}, \lambda\right) \in P_{\mathbb{U}}$,

$$
f\left(\lambda u_{1}+(1-\lambda) u_{2}\right) \leq[\geq] p\left(u_{1}, u_{2}, \lambda\right) f\left(u_{1}\right)+\left[1-p\left(u_{1}, u_{2}, \lambda\right)\right] f\left(u_{2}\right) .
$$

Then $f$ is called $p$-convex $[p$-concave $]$.

Definition 4. Let $T$ be a function defined on $Q_{\mathbb{U}}$. A function $f$ on $\mathbb{U}$ is called $T$-convex $[T$-concave $]$ if

$$
f\left(u_{2}\right)-f\left(u_{1}\right) \leq[\geq] T(\bar{u})\left[f\left(u_{3}\right)-f\left(u_{2}\right)\right] \quad \text { for } \bar{u} \in Q_{\mathbb{U}} .
$$

Now we give a simple characterization of poorly convex and poorly concave functions on $\mathbb{U}$. It gives an equivalence between poor convexity and $T$-convexity. In the special case $\mathbb{U}=[a, b] \subset \mathbb{R}$, this theorem has been proved in Radzik (2014) (see Proposition 1 there), and its proof is repeated with small changes.

THEOREM 1. A function $f$ on $\mathbb{U}$ is poorly convex [poorly concave] if and only if there is a positive function $T$ defined on $Q_{\mathbb{U}}$ such that $f$ is $T$-convex [T-concave]. 
Proof. $(\Rightarrow)$ Let $\left(u_{1}, u_{3}, \lambda\right) \in P_{\mathbb{U}}$ and let $u_{2}=\lambda u_{1}+(1-\lambda) u_{3}$. Then (5) (with $u_{2}$ replaced by $u_{3}$ ) implies that

$$
f\left(u_{2}\right) \leq[\geq] p\left(u_{1}, u_{3}, \lambda\right) f\left(u_{1}\right)+\left[1-p\left(u_{1}, u_{3}, \lambda\right)\right] f\left(u_{3}\right) .
$$

This is clearly equivalent to $(6)$ with

$$
T(\bar{u})=\frac{1-p\left(u_{1}, u_{3}, \lambda\right)}{p\left(u_{1}, u_{3}, \lambda\right)} .
$$

This equality uniquely defines a positive function $T$ on $Q_{\mathbb{U}}$, because any $\bar{u} \in Q_{\mathbb{U}}$ uniquely determines $0<\lambda<1$ (by the equality $u_{2}=\lambda u_{1}+(1-\lambda) u_{3}$ ) and $0<p<1$.

$(\Leftarrow)$ Let $\bar{u} \in Q_{\mathbb{U}}$. Then $u_{2}=\lambda u_{1}+(1-\lambda) u_{3}$ for a unique $0<\lambda<1$. If we put this value of $u_{2}$ in (6), and replace $u_{3}$ by $u_{2}$, we get

$$
\begin{aligned}
f\left(\lambda u_{1}\right. & \left.+(1-\lambda) u_{2}\right)-f\left(u_{1}\right) \\
& \leq[\geq] T\left(u_{1}, \lambda u_{1}+(1-\lambda) u_{2}, u_{2}\right)\left[f\left(u_{2}\right)-f\left(\lambda u_{1}+(1-\lambda) u_{2}\right)\right] .
\end{aligned}
$$

We can easily check that this inequality is equivalent to (5) with

$$
p\left(u_{1}, u_{2}, \lambda\right)=\frac{1}{1+T\left(u_{1}, \lambda u_{1}+(1-\lambda) u_{2}, u_{2}\right)} .
$$

This correctly describes a function $p$ on $P_{\mathbb{U}}$ as $T>0$, completing the proof.

It turns out that poorly convex functions have four simple properties, expressed in the next proposition. The first two of them are similar to properties of classical convex functions. The other two show that poor convexity is an intermediate property between convexity and quasi-convexity.

Proposition 2. For any functions $f$ and $g$ on $\mathbb{U}$, the following statements hold:

(a) $f$ is poorly convex if and only if $-f$ is poorly concave.

(b) If $f$ is poorly convex, then for every $\alpha \geq 0$ and $\beta \in \mathbb{R}$ the function $\alpha f+\beta$ is also poorly convex.

(c) If $f$ is convex, then it is poorly convex.

(d) If $f$ is poorly convex, then it is quasi-convex.

Proof. Statements (a) and (b) follow immediately from Definition 3. Statement (c) trivially follows from the fact that for convex functions inequality (5) holds for $p\left(u_{1}, u_{2}, \lambda\right) \equiv \lambda$.

Now, let $c \in \mathbb{R}$ and $u_{1}, u_{2} \in \mathbb{U}$ with $u_{1} \prec u_{2}$. Assume that $f\left(u_{1}\right) \leq c$ and $f\left(u_{2}\right) \leq c$. By assumption, $f$ satisfies (5) with " $\leq$ ". Therefore, for any $0<\lambda<1$ we have

$$
f\left(\lambda u_{1}+(1-\lambda) u_{2}\right) \leq p\left(u_{1}, u_{2}, \lambda\right) c+\left[1-p\left(u_{1}, u_{2}, \lambda\right)\right] c=c .
$$

Hence the set $\{u \in \mathbb{U}: f(u) \leq c\}$ is convex, so $f$ is quasi-convex. This proves $(\mathrm{d})$. 
REMARK 1. Obviously, the function $T$ corresponding to a poorly convex function $f$ described in Theorem 1 is not unique. For example, consider the convex function $f(u)=u^{2}$ with domain $[a, b](0 \leq a<b)$. One can easily see that for any function $T$ of the form $T(\bar{u})=k \cdot \frac{u_{2}-u_{1}}{u_{3}-u_{2}}$ for $\bar{u} \in Q_{[a, b]}$ (see $\sqrt{4}$ ) with $k \geq 1$, inequality (6) with " $\leq$ " holds for all $\bar{u} \in Q_{[a, b]}$. Hence, by (7) it follows that the function $p$ in Definition 3 is not unique either.

REMARK 2. Obviously, not every poorly convex function $f$ is convex. Moreover, there are strictly concave functions which are poorly convex. To see this, consider an interval $[a, b] \subset \mathbb{R}$ with $a<b$, and let $f$ be the strictly concave function $f(u)=-(u-a)^{2}$ on $\mathbb{U}=[a, b]$. Using (4), we easily see that the function $F(\bar{u})=\frac{f\left(u_{2}\right)-f\left(u_{1}\right)}{f\left(u_{3}\right)-f\left(u_{2}\right)}$ on $Q_{[a, b]}$ is positive and it trivially satisfies $(6) \leq$. Therefore, by Theorem $1, f$ is poorly convex.

On the other hand, not every quasi-convex function is poorly convex. To see this, consider the following continuous quasi-convex function on $[0,2]$ :

$$
f(u)= \begin{cases}u & \text { for } u \in[0,1), \\ 1 & \text { for } u \in[1,2],\end{cases}
$$

and suppose that it is poorly convex. This implies that $(6) \leq$ is satisfied for all $\bar{u} \in Q_{[0,2]}$. But putting $\bar{u}=(0,1,2)$ in $(6)$, we get $1-0 \leq \bar{T}(0,1,2) \cdot[1-1]$, which is impossible. A full characterization of poorly functions is given in Theorem 5 in the next section.

Now we give another definition:

Definition 5. A family $\mathcal{F}$ of functions on $\mathbb{U}$ is called pairwise poorly convex [concave] if for any two functions in $\mathcal{F}$ there is a positive function $p: P_{\mathbb{U}} \rightarrow(0,1)$ such that both functions are $p$-convex [ $p$-concave] on $\mathbb{U}$.

The above definition and the proof of Theorem 1 immediately lead to another characterization of pairwise poorly convex [concave] families of functions.

TheOREM 2. A family $\mathcal{F}$ of poorly convex [concave] functions on $\mathbb{U}$ is pairwise poorly convex [concave] if and only if for any two functions in $\mathcal{F}$ there is a positive function $T$ defined on $Q_{\mathbb{U}}$ such that both functions are $T$-convex $[T$-concave $]$ on $\mathbb{U}$.

Theorem 2 allows us to easily construct various pairwise poorly convex families of functions, as described in the following proposition.

Proposition 3. The following families of functions are pairwise poorly convex on $\mathbb{U}$ :

(a) any family of convex functions on $\mathbb{U}$;

(b) any family of the form $\mathcal{F}=\left\{\tilde{f}_{\alpha \beta}=\alpha+\beta \cdot f: \alpha \in \mathbb{R}, \beta \geq 0\right\}$, where $f$ is a fixed poorly convex function on $\mathbb{U}$; 
(c) any family of the form $\mathcal{G}=\left\{\tilde{g}_{\alpha \beta \gamma}=\alpha+\beta \cdot g_{\gamma}: \alpha \in \mathbb{R}, \beta \geq 0, \gamma \in \mathcal{A}\right\}$ for a fixed function $g_{\gamma}$ on $\mathbb{U}$, and a set $\mathcal{A}$ such that the family $\left\{g_{\gamma}\right\}_{\gamma \in \mathcal{A}}$ is pairwise poorly convex on $\mathbb{U}$.

Proof. Statement (a) is a consequence of the fact that all convex functions are $p$-convex with the same function $p$ of the form $p\left(u_{1}, u_{2}, \lambda\right) \equiv \lambda$ for $\left(u_{1}, u_{2}, \lambda\right) \in P_{\mathbb{U}}$. Statements (b) and (c) can be easily justified by using $(6) \leq$. The details are omitted.

REMARK 3. One can easily see that not every family of poorly convex functions is pairwise poorly convex. To see this, consider the family $\{g, h\}$ consisting of the two functions on $[-1,1]$ defined by $g(u)=-2 u^{2}+4 u+1$ and $h(u)=-2 u^{2}-4 u+1$. Let $T_{g}$ and $T_{h}$ be functions on $Q_{[-1,1]}$ defined by

$$
T_{g}(\bar{u})=\frac{g\left(u_{2}\right)-g\left(u_{1}\right)}{g\left(u_{3}\right)-g\left(u_{2}\right)} \quad \text { and } \quad T_{h}(\bar{u})=\frac{h\left(u_{2}\right)-h\left(u_{1}\right)}{h\left(u_{3}\right)-h\left(u_{2}\right)}
$$

for $\bar{u}=\left(u_{1}, u_{2}, u_{3}\right) \in Q_{[-1,1]}$. Since $g$ is strictly increasing and $h$ is strictly decreasing on $[-1,1]$, we have $T_{g}(\bar{u})>0$ and $T_{h}(\bar{u})>0$ for $\bar{u} \in Q_{[-1,1]}$. Moreover, inequality (6) $\leq$ trivially holds for both $f=g$ and $f=h$ with $T=T_{g}$ and $T=T_{h}$, respectively. Hence, by Theorem 1 the functions $g$ and $h$ are poorly convex. Suppose now that they are pairwise poorly convex with respect to a function $T$ on $Q_{[-1,1]}$. Then putting $u_{1}=-1, u_{2}=0$ and $u_{3}=1$ in $(6) \leq$ taken for $f=g$ and for $f=h$, we get

$$
1-(-5) \leq T(\bar{u})(3-1) \quad \text { and } \quad 1-3 \leq T(\bar{u})(-5-1) .
$$

Hence, $T(\bar{u}) \geq 3$ and $T(\bar{u}) \leq 1 / 3$, which is impossible. Therefore, the family $\{g, h\}$ is not pairwise poorly convex.

In the next two sections, necessary and sufficient conditions for two poorly convex functions to be also pairwise poorly convex are discussed. They can be used to construct various examples of pairwise poorly convex families consisting of nonconvex functions described by standard formulas (see Examples 3 and 4 in Radzik (2014) and Examples 2-4 in Section 6).

3. Poorly convex functions on intervals in $\mathbb{R}$. In this section we give a review of auxiliary results concerning poorly convex functions on an interval $[a, b]$ in $\mathbb{R}$. Some of them are proved in Radzik $(2014)$. These results will be used in the next section, where properties of poorly convex functions on a convex set $\mathbb{U} \subset \mathbb{R}^{n}$ are discussed.

We begin with a result (Proposition 4 in Radzik (2014)) showing that poor convexity on an interval in $\mathbb{R}$ is a very slight strengthening of quasiconvexity. 
THEOREM 3. Any continuous quasi-convex function on $[a, b]$ can be approximated by a sequence of poorly convex continuous functions in the topology of uniform convergence.

The next simple result gives a special formula for the function $T$ on $Q_{[a, b]}$ in (6) corresponding to a poorly convex function $f$ on $[a, b]$.

Let us define a function $T_{f}$ on $Q_{[a, b]}$ by

$$
T_{f}(\bar{u}):= \begin{cases}\frac{f\left(u_{2}\right)-f\left(u_{1}\right)}{f\left(u_{3}\right)-f\left(u_{2}\right)} & \text { if }\left[f\left(u_{2}\right)-f\left(u_{1}\right)\right]\left[f\left(u_{3}\right)-f\left(u_{2}\right)\right]>0, \\ 1 & \text { otherwise. }\end{cases}
$$

TheOREM 4. A function $f$ on $[a, b]$ is poorly convex if and only if it is $T_{f}$-convex.

Proof. $(\Rightarrow)$ Obviously, $T_{f}(\bar{u})>0$ for $\bar{u} \in Q_{[a, b]}$. By Theorem 1, there is a positive function $T$ on $Q_{[a, b]}$ such that $(6)_{\leq}$holds for all $\bar{u} \in Q_{[a, b]}$. But this implies that the system of inequalities $\left[f\left(u_{2}\right)-f\left(u_{1}\right)>0, f\left(u_{3}\right)-f\left(u_{2}\right)<0\right]$ does not hold (because $T>0$ ). Now, one can directly check that the function $T=T_{f}$ given by (8) satisfies $(6) \leq$ in all the remaining cases, and so $f$ is $T_{f}$-convex on $[a, b]$.

$(\Leftarrow)$ This is an immediate consequence of Theorem 1 .

The next theorem brings another simple characterization of an arbitrary poorly convex function on an interval in $\mathbb{R}$, given in terms of monotonicity properties. This theorem generalizes Proposition 3 from Radzik (2014), where this characterization is given for continuous poorly convex functions only.

For a function $f$ on $[a, b]$ we define

$$
\begin{aligned}
\tau_{f} & :=\sup \{x \in[a, b]: f \text { is strictly decreasing on }[a, x]\}, \\
\tau^{f} & :=\inf \{x \in[a, b]: f \text { is strictly increasing on }[x, b]\} .
\end{aligned}
$$

Obviously, $\tau_{f} \leq \tau^{f}$. Moreover, since every decreasing sequence in $\mathbb{R}$ is convergent (possibly to $-\infty$ ), one can easily see that the limits $f\left(\tau_{f}-\right)$ and $f\left(\tau^{f}+\right)$ exist if $a<\tau_{f}$ and $\tau^{f}<b$, respectively. Both the quantities $\tau_{f}$ and $\tau^{f}$ are basic for our subsequent considerations.

TheOrem 5. A function $f$ on $[a, b] \subset \mathbb{R}$ is poorly convex if and only if

(i) in case $\tau_{f}<\tau^{f}: f$ is strictly decreasing on $\left[a, \tau_{f}\right]$, strictly increasing on $\left[\tau^{f}, b\right]$, and constant on $\left(\tau_{f}, \tau^{f}\right)$ with value $\leq \min \left\{f\left(\tau_{f}\right), f\left(\tau^{f}\right)\right\}$

(ii) in case $\tau_{f}=\tau^{f}(:=\tau): f$ is strictly decreasing on $[a, \tau)$ and strictly increasing on $(\tau, b]$ with $f(\tau) \leq \max \{f(\tau-), f(\tau+)\}$. (Here we define $f(a-)=f(a)$ and $f(b+)=f(b)$. $)$

For the proof of the theorem we need the following lemma. 
Lemma 1. Let $f$ be a poorly convex function on $[a, b]$, and let $a \leq u_{1}<$ $u_{2} \leq b$.

(i) If $f\left(u_{1}\right)>f\left(u_{2}\right)$, then $f$ is strictly decreasing on $\left[a, u_{1}\right]$.

(ii) If $f\left(u_{1}\right)<f\left(u_{2}\right)$, then $f$ is strictly increasing on $\left[u_{2}, b\right]$.

Proof. Assume first that $f\left(u_{1}\right)>f\left(u_{2}\right)$. By Theorem $1, f$ is $T$-convex for some positive function $T$ defined on $Q_{[a, b]}$. Let $a \leq u^{\prime}<u^{\prime \prime}<u_{1}<u_{2} \leq b$ and denote $T_{1}:=T\left(u^{\prime}, u^{\prime \prime}, u_{1}\right)$ and $T_{2}:=T\left(u^{\prime \prime}, u_{1}, u_{2}\right)$. Then (6) implies that

$$
f\left(u^{\prime \prime}\right)-f\left(u^{\prime}\right) \leq T_{1}\left[f\left(u_{1}\right)-f\left(u^{\prime \prime}\right)\right] \leq T_{1} T_{2}\left[f\left(u_{2}\right)-f\left(u_{1}\right)\right],
$$

whence $f\left(u^{\prime}\right)>f\left(u^{\prime \prime}\right)$ and $f\left(u^{\prime \prime}\right)>f\left(u_{1}\right)$ because $f\left(u_{1}\right)>f\left(u_{2}\right)$ and $T_{1}, T_{2}>0$. This proves (i).

The proof of (ii) is analogous: starting from $f\left(u_{1}\right)<f\left(u_{2}\right)$, we repeat the previous reasoning, replacing $u^{\prime}, u^{\prime \prime}, u_{1}, u_{2}$ by $u_{1}, u_{2}, u^{\prime}, u^{\prime \prime}$, respectively.

Proof of Theorem 5. $(\Rightarrow)$ First assume that $\tau_{f}<\tau^{f}$. By (9) and (10), $f$ is strictly decreasing on $\left[a, \tau_{f}\right)$ and strictly increasing on $\left(\tau^{f}, b\right]$. Suppose that $f\left(\tau_{f}-\right)<f\left(\tau_{f}\right)$. Then there is an $u^{\prime \prime}$ with $a \leq u^{\prime \prime}<\tau_{f}$ such that $f\left(u^{\prime \prime}\right)<f\left(\tau_{f}\right)$. Hence Lemma 1 implies that $f$ is strictly increasing on $\left[\tau_{f}, b\right]$, which is impossible. Therefore $f\left(\tau_{f}-\right) \geq f\left(\tau_{f}\right)$, and consequently $f$ is strictly decreasing on $\left[a, \tau_{f}\right]$. In an analogous way we show that $f$ is strictly increasing on $\left[\tau^{f}, b\right]$.

Choose $u_{1}, u_{2}$ with $\tau_{f}<u_{1}<u_{2}<\tau^{f}$. If $f\left(u_{1}\right)>f\left(u_{2}\right)$ or $f\left(u_{1}\right)<f\left(u_{2}\right)$ then, by Lemma 1, $f$ is strictly decreasing on $\left[a, u_{1}\right]$ or strictly increasing on $\left[u_{2}, b\right]$, respectively. But this would contradict the definition of $\tau_{f}$ or $\tau^{f}$. Therefore $f\left(u_{1}\right)=f\left(u_{2}\right)$, and so $f \equiv C$ on $\left(\tau_{f}, \tau^{f}\right)$ for some constant $C$.

Now let $\tau_{f}<u^{\prime}<\tau^{f}$, whence $f\left(u^{\prime}\right)=C$. If $f\left(\tau_{f}\right)<f\left(u^{\prime}\right)$ or $f\left(\tau^{f}\right)<$ $f\left(u^{\prime}\right)$, then Lemma 1 implies that $f$ is strictly increasing on $\left[u^{\prime}, b\right]$ or strictly decreasing on $\left[a, u^{\prime}\right]$, respectively. But this, in view of $\tau_{f}<u^{\prime}<\tau^{f}$, would contradict the definition of $\tau_{f}$ or $\tau^{f}$, respectively. Therefore $f\left(\tau_{f}\right) \geq f\left(u^{\prime}\right)$ and $f\left(\tau^{f}\right) \geq f\left(u^{\prime}\right)$, completing the proof for $\tau_{f}<\tau^{f}$, whence $C \leq \min \left\{f\left(\tau_{f}\right)\right.$, $\left.f\left(\tau^{f}\right)\right\}$.

For (ii), in view of $(9)$ and $(10)$, it suffices to show that $f(\tau) \leq \max \{f(\tau-)$, $f(\tau+)\}$. Suppose on the contrary that $f(\tau)>\max \{f(\tau-), f(\tau+)\}$. Then there are $u^{\prime}<\tau<u^{\prime \prime}$ such that $f(\tau)-f\left(u^{\prime}\right)>0$ and $f\left(u^{\prime \prime}\right)-f(\tau)<0$. But this contradicts the inequality $f(\tau)-f\left(u^{\prime}\right) \leq T_{1}\left[f\left(u^{\prime \prime}\right)-f(\tau)\right]$ with some $T_{1}>0$, following from Theorem 11. This ends the proof of $(\Rightarrow)$.

$(\Leftarrow)$ We can directly verify (in both cases (i) and (ii)) that $f$ is $T_{f}$-convex for the function $T_{f}$ defined in (8). This, by Theorem 4 , completes the proof of Theorem 5 ,

As mentioned earlier, the version of Theorem 5 for $f$ continuous is given in Radzik (2014). The next theorem presents the same result for lower semicontinuous functions. It is very surprising that the formulation of both char- 
acterization theorems is identical and exceptionally simple. The lower semicontinuity of functions will be assumed in all the remaining sections of the paper.

THEOREM 6. Let $f$ be a lower semicontinuous function on $[a, b]$. Then $f$ is poorly convex if and only if there are $c \leq d$ in $[a, b]$ such that $f$ is strictly decreasing on $[a, c]$, strictly increasing on $[d, b]$, and constant on $[c, d]$.

Proof. This is an easy consequence of Theorem 5 and the property of lower semicontinuous functions described by Proposition 11(c). The details are omitted.

REMARK 4. It follows from Theorem 6 that a poorly convex function on an interval can be strictly concave (for example, $f(u)=-u^{2}$ on $[0,1]$ ). Moreover, every convex function on an interval $[a, b] \subset \mathbb{R}$ is continuous on $(a, b)$ and satisfies $f(a+) \leq f(a)$ and $f(b-) \leq f(b)$, which implies its upper semicontinuity on $[a, b]$. It is rather surprising that a poorly convex function on $[a, b]$ need not be either continuous on $(a, b)$ or even bounded from below on $(a, b)$. To see this, consider the function $f$ on $[-1,1]$ defined by

$$
f(u)= \begin{cases}1 / u & \text { for } u \in[-1,0), \\ -u^{2}+2 u & \text { for } u \in[0,1] .\end{cases}
$$

One can easily see that $\tau_{f}=\tau^{f}=0$. Hence, by Theorem $5, f$ is poorly convex on $[-1,1]$ and is neither continuous nor bounded above on $(-1,1)$.

The next three results (Theorems 7,9 give necessary and sufficient conditions for a pair of lower semicontinuous poorly convex functions on $[a, b]$ to be pairwise poorly convex. In Radzik (2014) these theorems have been stated for continuous functions (see Propositions 5-7 there), but one can check that the proofs work also for lower semicontinuous functions.

THEOREM 7. Let $f$ and $g$ be lower semicontinuous and poorly convex functions on $[a, b]$ satisfying $\left[\tau_{f}, \tau^{f}\right] \cap\left[\tau_{g}, \tau^{g}\right] \neq \emptyset$. Then the family $\{f, g\}$ is pairwise poorly convex on $[a, b]$.

The next theorem concerns the case $\left[\tau_{f}, \tau^{f}\right] \cap\left[\tau_{g}, \tau^{g}\right]=\emptyset$. To formulate it, we set

$$
Q_{g}^{f}:=\left\{\bar{u}: \tau^{f} \leq u_{1}<u_{2}<u_{3} \leq \tau_{g}\right\} .
$$

THEOREM 8. Let $f$ and $g$ be lower semicontinuous and poorly convex functions on $[a, b]$ with $\tau^{f}<\tau_{g}$. Then the family $\{f, g\}$ is pairwise poorly convex on $[a, b]$ if and only if

$$
\frac{f\left(u_{2}\right)-f\left(u_{1}\right)}{f\left(u_{3}\right)-f\left(u_{2}\right)} \leq \frac{g\left(u_{2}\right)-g\left(u_{1}\right)}{g\left(u_{3}\right)-g\left(u_{2}\right)} \quad \text { for } \bar{u} \in Q_{g}^{f} .
$$


The verification of (11) might be troublesome. It turns out that under a somewhat stronger assumption, (11) can be replaced by a much simpler condition.

THEOREM 9. Let $f$ and $g$ be lower semicontinuous and poorly convex functions on $[a, b]$ that are continuous on $\left[\tau^{f}, \tau_{g}\right]$ with $\tau^{f}<\tau_{g}$. Assume that there are continuous derivatives $f^{\prime}$ and $g^{\prime}$ on $\left(\tau^{f}, \tau_{g}\right)$, with $g^{\prime} \neq 0$. Then $\{f, g\}$ is pairwise poorly convex on $[a, b]$ if and only if $G=f^{\prime} / g^{\prime}$ is nonincreasing on $\left(\tau^{f}, \tau_{g}\right)$.

A classical property of a pair of convex functions $f_{1}$ and $f_{2}$ is that any linear combination $\alpha f_{1}+\beta f_{2}$ with $\alpha, \beta>0$ is also convex. Unfortunately, this is no longer the case for poorly convex functions. However, the following theorem holds:

TheOREM 10. Let $f_{1}$ and $f_{2}$ be poorly convex functions on $[a, b]$. Then $f=\alpha f_{1}+\beta f_{2}$ is poorly convex on $[a, b]$ for every $\alpha, \beta>0$ if and only if the family $\left\{f_{1}, f_{2}\right\}$ is pairwise poorly convex.

Proof. $(\Leftarrow)$ This implication is an easy consequence of Theorems 1 and 2 and Definition 5. Namely, we know that $(6) \leq$ (with some function $T$ on $[a, b])$ holds for both $f=f_{1}$ and $f=f_{2}$. But this trivially implies that this inequality also holds for any function $f=\alpha f_{1}+\beta f_{2}$ with $\alpha, \beta>0$, and so such an $f$ is poorly convex on $[a, b]$.

$(\Rightarrow)$ By Theorem 1, $f_{1}$ is $T_{1}$-convex and $f_{2}$ is $T_{2}$-convex on $[a, b]$ for some functions $T_{1}, T_{2}$ defined on $Q_{[a, b]}$. Further, by Theorem 1 , for any $\alpha, \beta>0$ there is a function $T_{\alpha \beta}$ on $Q_{[a, b]}$ such that $f=\alpha f_{1}+\beta f_{2}$ is $T_{\alpha \beta}$-convex on $[a, b]$.

Fix $\bar{u} \in Q_{[a, b]}$ and denote

$$
\begin{aligned}
& a_{1}:=f_{1}\left(u_{2}\right)-f_{1}\left(u_{1}\right), \quad b_{1}:=f_{1}\left(u_{3}\right)-f_{1}\left(u_{2}\right), \\
& a_{2}:=f_{2}\left(u_{2}\right)-f_{2}\left(u_{1}\right), \quad b_{2}:=f_{2}\left(u_{3}\right)-f_{2}\left(u_{2}\right), \\
& h_{1}:=T_{1}(\bar{u}), \quad h_{2}:=T_{2}(\bar{u}), \quad h_{\alpha \beta}:=T_{\alpha \beta}(\bar{u}) .
\end{aligned}
$$

Therefore, by Theorem 1 .

$$
h_{1}>0, \quad a_{1} \leq h_{1} b_{1}, \quad h_{2}>0, \quad a_{2} \leq h_{2} b_{2},
$$

and

$$
h_{\alpha \beta}>0, \quad \alpha a_{1}+\beta a_{2} \leq h_{\alpha \beta}\left(\alpha b_{1}+\beta b_{2}\right) \quad \text { for all } \alpha, \beta>0 .
$$

To complete the proof it suffices to show that $(12)$ and 130 imply the existence of some $h>0$ such that

$$
a_{1} \leq h b_{1} \quad \text { and } \quad a_{2} \leq h b_{2} .
$$

(Then we can define $T(\bar{u})=h$.)

We consider six cases. 
CAsE 1: $b_{1}=0$ or $b_{2}=0$. Then it follows from $(12)$ that $(14)$ is satisfied for $h=h_{2}$ or $h=h_{1}$, respectively.

CASE 2: $b_{1}>0$ and $h_{1} \leq h_{2}$. Then (12) implies that (14) is satisfied for $h=h_{2}$.

CASE 3: $b_{1}>0, h_{1}>h_{2}$ and $b_{2}>0$. Then, similarly to Case 2, (14) is satisfied for $h=h_{1}$.

CASE 4: $b_{1}>0, h_{1}>h_{2}$ and $b_{2}<0$. If $a_{1} \leq 0$ then $a_{1} \leq h_{2} b_{1}$ because $h_{2}>0$, and consequently (14) holds for $h=h_{2}$. Therefore we can assume that $a_{1}>0$.

On the other hand, $a_{2}<0$ as a consequence of the inequalities $a_{2} \leq$ $h_{2} b_{2}, h_{2}>0$ and $b_{2}<0$. Hence, $h_{1}^{\prime}:=a_{1} / b_{1}>0, h_{2}^{\prime}:=a_{2} / b_{2}>0$, and $a_{1}=h_{1}^{\prime} b_{1}$ and $a_{2}=h_{2}^{\prime} b_{2}$. But this implies that if $h_{1}^{\prime} \leq h_{2}^{\prime}$ then $a_{1} \leq h_{2}^{\prime} b_{1}$, and consequently $(14)$ is satisfied for $h=h_{2}^{\prime}$.

Now consider the second subcase, $h_{1}^{\prime}>h_{2}^{\prime}$, that is, $a_{1} / b_{1}>a_{2} / b_{2}$. Since the last inequality implies that $0<-a_{2} / a_{1}<-b_{2} / b_{1}$ (because $a_{1}>0$, $a_{2}<0, b_{1}>0$ and $\left.b_{2}<0\right)$, we can find $\alpha_{0}, \beta_{0}>0$ such that $-a_{2} / a_{1}<$ $\alpha_{0} / \beta_{0}<-b_{2} / b_{1}$, or equivalently $\alpha_{0} a_{1}+\beta_{0} a_{2}>0>\alpha_{0} b_{1}+\beta_{0} b_{2}$. But this contradicts the inequality in (13) for $\alpha=\alpha_{0}$ and $\beta=\beta_{0}$. So the subcase $h_{1}^{\prime}>h_{2}^{\prime}$ is impossible. Therefore, also in Case 4 there is an $h>0$ for which (14) holds.

CASE 5: $b_{1}<0$ and $b_{2} \leq 0$. Then it is easily seen that (14) holds for $h=\min \left\{h_{1}, h_{2}\right\}$.

CASE 6: $b_{1}<0$ and $b_{2}>0$. Here we can consider two subcases, $h_{2} \leq h_{1}$ and $h_{2}>h_{1}$, which can be analyzed in exactly the same way as in Cases 2 and 4 , after changing parameters $a_{1}, b_{1}, a_{2}, b_{2}, h_{1}, h_{2}$ there to $a_{2}, b_{2}, a_{1}, b_{1}$, $h_{2}, h_{1}$, respectively.

Thus the proof of Theorem 10 is complete.

REMARK 5. In view of Theorem 10, it is natural to ask whether the following general statement holds: Let $f_{1}, \ldots, f_{n}(n \geq 3)$ be poorly convex functions on $[a, b]$. Then $f=\sum_{i=1}^{n} \alpha_{i} f_{i}$ is poorly convex on $[a, b]$ for all $\alpha_{1}, \ldots, \alpha_{n}>0$ if and only if $\left\{f_{1}, \ldots, f_{n}\right\}$ is a family of pairwise poorly convex functions. Unfortunately, the author does not know the answer to this question.

4. Poorly convex functions on subsets of $\mathbb{R}^{n}$. In this section, relying on the results of the previous section, we analyze the main properties of poorly convex functions defined on convex subsets of $\mathbb{R}^{n}$. Theorems 11,13 below are basic for the characterization of pairwise poorly convex families of functions discussed in the next section. Here $Q_{[0,1]}$ is the subset of $[0,1]^{3}$ 
defined by (4), and $\mathbb{U}$ denotes any fixed convex subset of $\mathbb{R}^{n}$. We begin with a definition and an auxiliary lemma.

Definition 6. Let $f$ be a real function on $\mathbb{U}$, and let $v, w \in \mathbb{U}$. Then the function $f_{v}^{w}$ on $[0,1]$ defined by

$$
f_{v}^{w}(x)=f((1-x) v+x w), \quad 0 \leq x \leq 1,
$$

is called the cross function of $f($ at $(v, w))$.

THEOREM 11. A function $f$ on $\mathbb{U}$ is poorly convex if and only if for any $v, w \in \mathbb{U}$ with $v \prec w$ the cross function $f_{v}^{w}$ of $f$ is poorly convex on $[0,1]$.

Proof. $(\Rightarrow)$ Fix $\bar{x}=\left(x_{1}, x_{2}, x_{3}\right) \in Q_{[0,1]}$ and $v, w \in \mathbb{U}$ with $v \prec w$, and let $\bar{u}=\left(u_{1}, u_{2}, u_{3}\right)$ be defined by

$$
u_{i}=\left(1-x_{i}\right) v+x_{i} w \quad \text { for } i=1,2,3 .
$$

Then $u_{2}=\lambda u_{1}+(1-\lambda) u_{3}$ with $\lambda=\frac{x_{3}-x_{2}}{x_{3}-x_{1}}$, whence $0<\lambda<1$ and

$$
v \preceq u_{1} \prec u_{2} \prec u_{3} \preceq w,
$$

since $v \prec w$ and $0 \leq x_{1}<x_{2}<x_{3} \leq 1$. Therefore $\bar{u} \in Q_{\mathbb{U}}$.

By Theorem 1, there is a function $T: Q_{\mathbb{U}} \rightarrow \mathbb{R}$ such that $(6) \leq$ holds. By (16) and 15), this leads to

$$
f_{v}^{w}\left(x_{2}\right)-f_{v}^{w}\left(x_{1}\right) \leq \tilde{T}_{v}^{w}(\bar{x})\left[f_{v}^{w}\left(x_{3}\right)-f_{v}^{w}\left(x_{2}\right)\right],
$$

where $\tilde{T}_{v}^{w}(\bar{x})=T(\bar{u})>0$. Hence, Theorem 1 with $f=f_{v}^{w}$ and $\mathbb{U}=[0,1]$ implies that $f_{v}^{w}$ is poorly convex on $[0,1]$.

$(\Leftarrow)$ Assume that $f_{v}^{w}$ is poorly convex on $[0,1]$ for any $v, w \in \mathbb{U}$ with $v \prec w$. Then, by Theorem 1 with $f=f_{v}^{w}$ and $\mathbb{U}=[0,1]$, there is a positive function $\tilde{T}_{v}^{w}: Q_{[0,1]} \rightarrow \mathbb{R}$ such that 18 holds for all $\bar{x} \in Q_{[0,1]}$.

Fix $\bar{u}=\left(u_{1}, u_{2}, u_{3}\right) \in Q_{\mathbb{U}}$ with $u_{1}=v, u_{3}=w$ and $u_{2}=\lambda u_{1}+(1-\lambda) u_{3}$ for some $0<\lambda<1$. Then $\bar{x}=\left(x_{1}, x_{2}, x_{3}\right)=(0, \lambda, 1) \in Q_{[0,1]}$, and $\sqrt{16}$ and (18) hold. Now taking into account (15) and (16) we can easily check that (18) can be rewritten in the form (6) with $T(\bar{u}):=\tilde{T}_{v}^{w}(\bar{x})$. Hence, in view of $T_{v}^{w}(\bar{x})>0$ for $\bar{x} \in Q_{[0,1]}$, we can apply Theorem 1 again to conclude that $f$ is poorly convex on $\mathbb{U}$.

THEOREM 12. The family $\{g, h\}$ of two functions on $\mathbb{U}$ is pairwise poorly convex on $\mathbb{U}$ if and only if for any $v, w \in \mathbb{U}$ with $v \prec w$ the pair $\left\{g_{v}^{w}, h_{v}^{w}\right\}$ is pairwise poorly convex on $[0,1]$.

Proof. $(\Rightarrow)$ Fix $v, w \in \mathbb{U}$ with $v \prec w$, and let $\bar{x}=\left(x_{1}, x_{2}, x_{3}\right) \in Q_{[0,1]}$. Define $u_{1}, u_{2}, u_{3}$ by (16). Obviously (17) holds, because $\bar{x} \in Q_{[0,1]}$.

By Theorem 2, there is a function $T: Q_{\mathbb{U}} \rightarrow \mathbb{R}$ such that $(6) \leq$ holds for $f=g$ and $f=h$. Together with (16) and (15) this leads to

$$
\begin{aligned}
& g_{v}^{w}\left(x_{2}\right)-g_{v}^{w}\left(x_{1}\right) \leq \tilde{T}_{v}^{w}(\bar{x})\left[g_{v}^{w}\left(x_{3}\right)-g_{v}^{w}\left(x_{2}\right)\right], \\
& h_{v}^{w}\left(x_{2}\right)-h_{v}^{w}\left(x_{1}\right) \leq \tilde{T}_{v}^{w}(\bar{x})\left[h_{v}^{w}\left(x_{3}\right)-h_{v}^{w}\left(x_{2}\right)\right],
\end{aligned}
$$


with $\tilde{T}_{v}^{w}(\bar{x})=T(\bar{u})>0$. Hence, Theorem 2 taken for $\mathbb{U}=[0,1]$ and $f=g_{v}^{w}$ and $f=h_{v}^{w}$ immediately implies that $\left\{g_{v}^{w}, h_{v}^{w}\right\}$ is pairwise poorly convex on $[0,1]$ for all $v, w \in \mathbb{U}$.

$(\Leftarrow)$ Assume that $g_{v}^{w}, h_{v}^{w}$ are poorly convex on $[0,1]$ for any $v, w \in \mathbb{U}$ with $v \prec w$. Then, by Theorem 1 for $f=g_{v}^{w}, f=h_{v}^{w}$ and $\mathbb{U}=[0,1]$, there is a positive function $\tilde{T}_{v}^{w}: Q_{[0,1]} \rightarrow \mathbb{R}$ such that $\sqrt{19}$ and 20 hold for all $\bar{x} \in Q_{[0,1]}$.

Let $\bar{u}=\left(u_{1}, u_{2}, u_{3}\right) \in Q_{\mathbb{U}}$ with $u_{1}=v, u_{3}=w$ and with $u_{2}=\lambda u_{1}+(1-$ $\lambda) u_{3}$ for some $0<\lambda<1$. Then $\bar{x}=\left(x_{1}, x_{2}, x_{3}\right)=(0, \lambda, 1) \in Q_{[0,1]}$, and $(19)$ and (20) hold. But those two inequalities are equivalent (by (15) and (16) to (6) taken for $f=g$ and $f=h$, with the positive function $T$ defined by $T(\bar{u}):=\tilde{T}_{v}^{w}(\bar{x})$ for $\bar{u} \in Q_{\mathbb{U}}$. This proves that $\{g, h\}$ is pairwise poorly convex on $\mathbb{U}$.

REMARK 6. When $\mathbb{U}$ is a compact convex subset of $\mathbb{R}^{n}$, Theorems 11 and 12 can be simplified by replacing "for any $v, w \in \mathbb{U}$ with $v \prec w$ " with "for any $v, w \in \partial \mathbb{U}$ with $v \prec w$ " (here $\partial \mathbb{U}$ is the boundary of $\mathbb{U}$ ). This is an easy consequence of the fact that for all $v, w \in \mathbb{U}$ with $v \prec w$ there are $v^{\prime}, w^{\prime} \in \partial \mathbb{U}$ such that $v^{\prime} \preceq v \prec w \preceq w^{\prime}$ with $v=(1-x) v^{\prime}+x w^{\prime}$ and $w=(1-y) v^{\prime}+y w^{\prime}$ for some $0 \leq x, y<1$. The easy details are omitted.

THEOREM 13. Let $g$ and $h$ be poorly convex functions on $\mathbb{U}$. Then the function $f=\alpha g+\beta h$ is poorly convex for all $\alpha, \beta>0$ on $\mathbb{U}$ if and only if $\{g, h\}$ is pairwise poorly convex on $\mathbb{U}$.

Proof. $(\Leftarrow)$ This follows immediately from Theorem 2. Namely, if $(6) \leq$ holds for both $f=g$ and $f=h$, then it obviously holds for any $f=\alpha g+\beta \bar{h}$ with $\alpha, \beta>0$.

$(\Rightarrow)$ Assume that $f=\alpha g+\beta h$ is poorly convex for all $\alpha, \beta>0$ on $\mathbb{U}$, and fix $v, w \in \mathbb{U}$ with $v \prec w$. Let $g_{v}^{w}, h_{v}^{w}, f_{v}^{w}$ be the cross functions of $g, h, f$, respectively. Obviously $f_{v}^{w}(x)=\alpha g_{v}^{w}(x)+\beta h_{v}^{w}(x)$ for all $x \in[0,1]$ and $\alpha, \beta>0$. Therefore, by Theorem 11, $f_{v}^{w}$ is poorly convex on $[0,1]$ for all $\alpha, \beta>0$. Consequently, Theorem 10 implies that $\left\{g_{v}^{w}, h_{v}^{w}\right\}$ is pairwise poorly convex on $[0,1]$. Hence, Theorem 12 yields the pairwise poor convexity of $\{g, h\}$ on $\mathbb{U}$.

5. Main theorem on pairwise poorly convex families. In the previous sections the theory of lower semicontinuous and poorly convex functions was developed. Theorem 14 in this section completes our considerations about such functions; it will be applied to some optimization problems in Section 7. Before stating it, we quote two classical results from the literature (Eckhoff (1993), Sion (1958)). 
Proposition 4 (Helly's intersection theorem). Let $\mathcal{M}$ be a family of at least $n+1$ convex sets in $\mathbb{R}^{n}$ such that $\mathcal{M}$ is finite or each member of $\mathcal{M}$ is compact. Then if any $n+1$ members of $\mathcal{M}$ have a common point, there is a point common to all members of $\mathcal{M}$.

Proposition 5 (Sion's minimax theorem). Let $X$ and $Y$ be convex subsets of linear topological spaces with $X$ compact. Let $f: X \times Y \rightarrow \mathbb{R}$ be such that $f(\cdot, y)$ is upper semicontinuous and quasi-concave for each $y \in Y$, and $f(x, \cdot)$ is lower semicontinuous and quasi-convex for each $x \in X$. Then

$$
\sup _{x \in X} \inf _{y \in Y} f(x, y)=\inf _{y \in Y} \sup _{x \in X} f(x, y) .
$$

TheOREM 14. Let $\mathcal{T}=\left\{f_{\alpha}\right\}$ be a pairwise poorly convex family of lower semicontinuous functions on a compact convex set $\mathbb{U} \subset \mathbb{R}^{n}$. Suppose $\sup _{\alpha} f_{\alpha}(u)>0$ for each $u \in \mathbb{U}$. Then there are functions $f_{\alpha_{1}}, \ldots, f_{\alpha_{n+1}}$ in $\mathcal{T}$ and a vector $\left(\lambda_{1}, \ldots, \lambda_{n+1}\right)$ with nonnegative components satisfying $\sum_{i=1}^{n+1} \lambda_{i}=1$ such that

$$
\sum_{i=1}^{n+1} \lambda_{i} f_{\alpha_{i}}(u)>0 \quad \text { for all } u \in \mathbb{U} .
$$

REMARK 7. Since any family of convex functions is pairwise poorly convex (see Proposition 3(a)), Theorem 14 is a generalization of the result of Bohnenblust et al. (1950), where it is assumed that $\mathcal{T}$ consists of convex continuous functions on $\mathbb{U}$. Another special case of Theorem 14 for $n=1$ with a pairwise poorly convex family of continuous functions was earlier proved in Radzik (2014), where this result was used to get the existence of Nash equilibria with a very simple structure in several two-person nonzero-sum games on the unit square.

The proof of Theorem 14 is preceded by the following lemma.

Lemma 2. Let $\mathcal{T}=\left\{f_{1}, \ldots, f_{m}\right\}$ be a pairwise poorly convex [concave] family of functions defined on a convex compact subset $Z$ of $\mathbb{R}^{s}$, where $m \geq 2$ and $s \geq 1$. Then for any $\gamma_{1}, \ldots, \gamma_{m} \geq 0$ the function $K=\sum_{i=1}^{m} \gamma_{i} f_{i}$ is quasi-convex [quasi-concave] on $Z$.

Proof. In view of Proposition 1(a), it suffices to consider the "convex" case.

Fix any $\gamma_{1}, \ldots, \gamma_{m} \geq 0$ and define $Q_{Z}$ analogously to (2). Firstly we will prove that there is a positive function $T$ on $Q_{Z}$ such that all the functions $f_{1}, \ldots, f_{m}$ are $T$-convex on $Z$.

Fix $\bar{u}=\left(u_{1}, u_{2}, u_{3}\right)$ in $Q_{Z}$. Since each $f_{i}, 1 \leq i \leq m$, is poorly convex, Theorem 1 and (6) imply that it suffices to show that there is an $h>0$ such that

$$
f_{i}\left(u_{2}\right)-f_{i}\left(u_{1}\right) \leq h\left[f_{i}\left(u_{3}\right)-f_{i}\left(u_{2}\right)\right], \quad i=1, \ldots, m .
$$


Now, let $1 \leq k \leq m$. By poor convexity of $f_{k}$, there is an $h_{k}>0$ such that

$$
f_{k}\left(u_{2}\right)-f_{k}\left(u_{1}\right) \leq h_{k}\left[f_{k}\left(u_{3}\right)-f_{k}\left(u_{2}\right)\right] .
$$

One can easily see that if 23 holds for two positive numbers $h_{k}=h_{k}^{\prime}$ and $h_{k}=h_{k}^{\prime \prime}$, then it also holds for every convex combination $h_{k}=\alpha h_{k}^{\prime}+(1-\alpha) h_{k}^{\prime \prime}$, $0 \leq \alpha \leq 1$. Hence, all the sets

$$
A_{k}=\left\{h_{k}: h_{k}>0 \text { and } 23 \text { holds }\right\}, \quad k=1, \ldots, m,
$$

are nonempty convex subsets of $\mathbb{R}$. Now, from the pairwise poor convexity of $\mathcal{T}$, we easily conclude that any two of the sets $A_{i}$ have a common point. Hence, by Helly's intersection theorem, $\bigcap_{i=1}^{m} A_{i} \neq \emptyset$. Therefore there is an $h>0$ such that $(22)$ holds. Hence $f_{1}, \ldots, f_{m}$ are $T$-convex on $Z$ for some positive function $T$ on $Q_{Z}$.

So inequality $(6)<$ holds for $f=f_{1}, \ldots, f_{m}$. Hence, we easily see that it also holds for $K=\sum_{i=1}^{m} \gamma_{i} f_{i}$. Thus $K$ is poorly convex on $Z$, and therefore also quasi-convex on $Z$ (see Proposition 2 (d)).

Proof of Theorem 14. Since each $f_{\alpha}$ in $\mathcal{T}$ is lower semicontinuous on $\mathbb{U}$, the set

$$
U_{\alpha}=\left\{u \in \mathbb{U}: f_{\alpha}(u) \leq 0\right\}
$$

is closed, and hence compact. Moreover, $U_{\alpha}$ is also convex because $f_{\alpha}$ is quasi-convex (by Proposition 2(d) and Definition 1).

By the assumption, $\bigcap_{\alpha} U_{\alpha}=\emptyset$. Hence, by Helly's intersection theorem, there is a subfamily $\mathcal{T}^{\prime}=\left\{f_{\alpha_{1}}, \ldots, f_{\alpha_{n+1}}\right\}$ of $\mathcal{T}$ such that $\bigcap_{i=1}^{n+1} U_{\alpha_{i}}=\emptyset$. But this implies that

$$
\sup _{1 \leq i \leq n+1} f_{\alpha_{i}}(u)>0 \quad \text { for each } u \in \mathbb{U} \text {. }
$$

Let $\boldsymbol{x}:=\left(x_{1}, \ldots, x_{n+1}\right)$ and define a compact convex set $\mathcal{X} \subset \mathbb{R}^{n+1}$ by

$$
\mathcal{X}=\left\{\boldsymbol{x} \in \mathbb{R}^{n+1}: \sum_{i=1}^{n+1} x_{i}=1 \text { and } x_{1}, \ldots, x_{n+1} \geq 0\right\} .
$$

Let $F$ be the function on $\mathcal{X} \times \mathbb{U}$ defined by

$$
F(\boldsymbol{x}, u)=\sum_{i=1}^{n+1} x_{i} f_{\alpha_{i}}(u) .
$$

Obviously $F(\cdot, u)$ is linear and so continuous for every $u \in \mathbb{U}$. Hence, by Proposition 1 (a), for every $u \in \mathbb{U}$ the function $F(\cdot, u)$ is upper semicontinuous, quasi-convex and quasi-concave.

On the other hand, Proposition 1(b) implies that $F(\boldsymbol{x}, \cdot)$ is lower semicontinuous for every $\boldsymbol{x} \in \mathcal{X}$. Moreover, Lemma 2 implies that $F(\boldsymbol{x}, \cdot)$ is quasi-convex for every $\boldsymbol{x} \in \mathcal{X}$. 
So, by Sion's minimax theorem with $X=\mathcal{X}, Y=\mathbb{U}$ and $f=F$, there is a number $v \in \mathbb{R}$ such that

$$
\sup _{\boldsymbol{x} \in \mathcal{X}} \inf _{u \in \mathbb{U}} F(\boldsymbol{x}, u)=v=\inf _{u \in \mathbb{U}} \sup _{\boldsymbol{x} \in \mathcal{X}} F(\boldsymbol{x}, u) .
$$

Because both $\mathcal{X}$ and $\mathbb{U}$ are compact, we easily conclude the proof as follows: As stated before, the function $F(\boldsymbol{x}, u)$ is upper semicontinuous in $\boldsymbol{x}$ and lower semicontinuous in $u$. Hence, by Proposition 1(e), the functions $F_{1}(\boldsymbol{x})=\inf _{u \in \mathbb{U}} F(\boldsymbol{x}, u)$ on $\mathcal{X}$ and $F_{2}(u)=\sup _{\boldsymbol{x} \in \mathcal{X}} F(\boldsymbol{x}, u)$ on $\mathbb{U}$ are upper and lower semicontinuous, respectively. Therefore, by Proposition 1(d), 25) is equivalent to

$$
\max _{\boldsymbol{x} \in \mathcal{X}} \min _{u \in \mathbb{U}} F(\boldsymbol{x}, u)=v=\min _{u \in \mathbb{U}} \max _{\boldsymbol{x} \in \mathcal{X}} F(\boldsymbol{x}, u) .
$$

The first equality in 26 implies that there is a vector $\left(\lambda_{1}, \ldots, \lambda_{n+1}\right) \in \mathcal{X}$ such that

$$
\sum_{i=1}^{n+1} \lambda_{i} f_{\alpha_{i}}(u) \geq v \quad \text { for all } u \in \mathbb{U} .
$$

The second equality in 26) and Proposition 1(d) imply that there is a $u^{0} \in \mathbb{U}$ such that

$$
v=\min _{u \in \mathbb{U}} \max _{\boldsymbol{x} \in \mathcal{X}} F(\boldsymbol{x}, u)=\max _{\boldsymbol{x} \in \mathcal{X}} \sum_{i=1}^{n+1} x_{i} f_{\alpha_{i}}\left(u^{0}\right)>0
$$

because of (24). Comparing this with (27), we finally get 21).

REMARK 8. One could ask whether Theorem 14 remains true with "pairwise" cancelled. We can easily show that it does not: On $\mathbb{U}=[-1,1]$, define $f_{1}(u)=-2 u^{2}+4 u+1$ and $f_{2}(u)=-2 u^{2}-4 u+1$. It is shown in Remark 3 (Section 2) that these two functions are poorly convex but not pairwise poorly convex. Moreover, $\max \left\{f_{1}(u), f_{2}(u)\right\}>0$ for each $u \in[-1,1]$. Now, let $F_{\lambda}(u)=\lambda f_{1}(u)+(1-\lambda) f_{2}(u)$. One can directly calculate that $F_{\lambda}(-1)+F_{\lambda}(1)=-2$ for every $\lambda \in[0,1]$, and so at least one of the values $F_{\lambda}(-1)$ or $F_{\lambda}(1)$ is negative, so the conclusion of Theorem 14 does not hold.

6. Examples of pairwise poorly convex families. In this section we give four examples of pairwise poorly convex families described by rather standard formulas. Theorems 6, 9, 11] and 12 will be useful in analysing such families. In this section, $\mathbb{R}_{+}^{n}$ denotes the set of all vectors in $\mathbb{R}^{n}$ with nonnegative components. We also write $\bar{x}=\left(x_{1}, \ldots, x_{n}\right)$, and analogously for $\bar{a}, \bar{\alpha}$ etc.

EXAMPLE 1. Let $\bar{c} \in \mathbb{R}_{+}^{n}$ and consider the function

$$
f_{\bar{\alpha}}(\bar{x})=\sum_{i=1}^{n} c_{i}\left(x_{i}-\alpha_{i}\right)^{2}
$$


on a convex subset $\mathbb{U}$ of $\mathbb{R}^{n}$. Obviously, $f_{\bar{\alpha}}$ is convex on $\mathbb{U}$ for any $\bar{\alpha} \in \mathbb{R}^{n}$. Therefore, by Proposition $3(\mathrm{a}), \mathcal{F}=\left\{f_{\bar{\alpha}}: \bar{\alpha} \in \mathbb{R}^{n}\right\}$ is a pairwise poorly convex family.

ExAmple 2. Let $A>1, \bar{b} \in \mathbb{R}_{+}^{n}$ and let $\mathbb{U}$ be a convex subset of $\mathbb{R}^{n}$. Consider the family $\mathcal{G}=\left\{g_{\beta \gamma}: \beta \in \mathbb{R}, \gamma \in \mathbb{R}_{+}\right\}$of continuous functions $g_{\beta \gamma}$ on $\mathbb{U}$ defined by

$$
g_{\beta \gamma}(\bar{x})=\beta-\gamma \cdot A^{-\sum_{i=1}^{n} b_{i} x_{i}^{2}} .
$$

We will show that $\mathcal{G}$ is a pairwise poorly convex family.

In view of Proposition $3(\mathrm{~b})$, it suffices to show that $f(\bar{x})=-A^{-\sum_{i=1}^{n} b_{i} x_{i}^{2}}$ is poorly convex on $\mathbb{U}$. In general, $f$ need not be convex. For example, when $\mathbb{U}$ contains the interval connecting $(0, \ldots, 0)$ and $(B, 0, \ldots, 0)$ with $B=$ $\sqrt{1 /\left(b_{1} \ln A\right)}$, then the function $h(t)=-A^{-b_{1} t^{2}}$ is not convex on $[0, B]$, and so $f$ is not convex on $\mathbb{U}$.

Fix $v, w \in \mathbb{U}$ with $v=\left(v_{1}, \ldots, v_{n}\right), w=\left(w_{1}, \ldots, w_{n}\right)$ and $v \prec w$. Then the cross function $G(t)=g_{v}^{w}(t)$ (see 15) is a continuous function given by

$$
G(t)=-A^{-\sum_{i=1}^{n} b_{i}\left[(1-t) v_{i}+t w_{i}\right]^{2}}, \quad 0 \leq t \leq 1 \text {. }
$$

We have

$$
G^{\prime}(t)=-2 \ln A \cdot G(t) \cdot\left\{t \sum_{i=1}^{n} b_{i}\left(w_{i}-v_{i}\right)^{2}+\sum_{i=1}^{n} b_{i} v_{i}\left(w_{i}-v_{i}\right)\right\} .
$$

One can easily see that there is an $a \in[0,1]$ such that $G^{\prime}(t)<0$ for $t \in[0, a)$ and $G^{\prime}(t)>0$ for $t \in(a, 1]$. Consequently, $G$ is strictly decreasing in $[0, a]$ and strictly increasing in $[a, 1]$, which, by Theorems 6 and 11 , proves that $f$ is poorly convex on $\mathbb{U}$. Thus $\mathcal{G}$ is a pairwise poorly convex family.

ExAmple 3. Let $\mathbb{U}=\left\{\bar{x} \in \mathbb{R}^{n}: \pi / 2 \leq \sum_{i=1}^{n} x_{i} \leq 3 \pi / 2\right\}$. Consider the family $\mathcal{F}=\left\{f_{\alpha \beta \gamma}: \alpha \in \mathbb{R}, \beta>0,|\gamma| \leq \pi / 2\right\}$ of continuous functions $f_{\alpha \beta \gamma}$ on $\mathbb{U}$ given by

$$
f_{\alpha \beta \gamma}(\bar{x})=\alpha+\beta \cdot \cos \left\{\sum_{i=1}^{n} x_{i}-\gamma\right\} .
$$

We will show that $\mathcal{F}$ is a pairwise poorly convex family on $\mathbb{U}$.

Let $\mathcal{G}=\left\{g_{\gamma}:|\gamma| \leq \pi / 2\right\}$ be the subfamily of $\mathcal{F}$ consisting of the functions

$$
g_{\gamma}(\bar{x})=\cos \left\{\sum_{i=1}^{n} x_{i}-\gamma\right\} \text {. }
$$

By Proposition 3(c), we only need to show that $\mathcal{G}$ is a pairwise poorly convex family on $\mathbb{U}$.

It is easy to see that for $0<|\gamma| \leq \pi / 2$ the function $g(t):=g_{\gamma}(t, 0, \ldots, 0)$ $=\cos t$ is not convex on $[\pi / 2,3 \pi / 2]$. Consequently, the functions $g_{\gamma}$ (with 
$\gamma \in[-\pi / 2,0) \cup(0, \pi / 2])$ are not convex on $\mathbb{U}$, so Proposition 3(a) cannot be applied here.

Let $g_{\gamma} \in \mathcal{G}$ and fix $v, w \in \mathbb{U}$ with $v=\left(v_{1}, \ldots, v_{n}\right), w=\left(w_{1}, \ldots, w_{n}\right)$ and $v \prec w$. Then

$$
\pi / 2 \leq(1-t) \sum_{i=1}^{n} v_{i}+t \sum_{i=1}^{n} w_{i} \leq 3 \pi / 2 \quad \text { for } t \in[0,1],
$$

and the cross function $G_{\gamma}(t)=\left(g_{\gamma}\right)_{v}^{w}(t)$ is the continuous function

$$
G_{\gamma}(t)=\cos \left\{(1-t) \sum_{i=1}^{n} v_{i}+t \sum_{i=1}^{n} w_{i}-\gamma\right\}, \quad 0 \leq t \leq 1 .
$$

By Theorem 12, to show the pairwise poor convexity of $\mathcal{G}$ we need to show the same for $\mathcal{G}^{*}:=\left\{G_{\gamma}:-\pi / 2 \leq \gamma \leq \pi / 2\right\}$.

If $\sum_{i=1}^{n} v_{i}=\sum_{i=1}^{n} w_{i}$, then every $G_{\gamma}$ is a constant function, and then $\mathcal{G}^{*}$ is obviously pairwise poorly convex.

Without loss of generality, we can now assume that

$$
\sum_{i=1}^{n} v_{i}<\sum_{i=1}^{n} w_{i}
$$

This is an easy consequence of the fact (following from Theorem 6 ) that $G_{\gamma}$ is poorly convex on $[0,1]$ if and only if the function $f(t)=G_{\gamma}(1-t)$ is poorly convex on $[0,1]$.

Let $\tau_{\gamma}$ and $\tau^{\gamma}$ denote the quantities $\tau_{f}$ and $\tau^{f}$ (see $(9)$ and $(10)$ ) for $f=G_{\gamma}$, respectively. A simple analysis of the function $G_{\gamma}$ (in the context of inequalities (28), 29) and $|\gamma| \leq \pi / 2)$ implies that $\tau_{\gamma}=\tau^{\gamma}(=: \tau(\gamma))$ and

$$
\tau(\gamma)= \begin{cases}0 & \text { if } \sum_{i=1}^{n} v_{i} \geq \pi+\gamma \\ 1 & \text { if } \sum_{i=1}^{n} w_{i} \leq \pi+\gamma \\ \frac{\pi+\gamma-\sum_{i=1}^{n} v_{i}}{\sum_{i=1}^{n}\left(w_{i}-v_{i}\right)} & \text { if } \sum_{i=1}^{n} v_{i}<\pi+\gamma<\sum_{i=1}^{n} w_{i} .\end{cases}
$$

One can easily verify that $\tau(\gamma) \in[0,1]$ for all $\gamma \in[0,1], \tau\left(\gamma_{1}\right) \leq \tau\left(\gamma_{2}\right)$ if $\gamma_{1}<\gamma_{2}$, and $G_{\gamma}$ is strictly decreasing and strictly increasing in $[0, \tau(\gamma)]$ and $[\tau(\gamma), 1]$, respectively. Therefore, by Theorem $6, G_{\gamma}$ is poorly convex on $[0,1]$.

Now fix $-\pi / 2 \leq \gamma_{1}<\gamma_{2} \leq \pi / 2$. Then $\tau\left(\gamma_{1}\right) \leq \tau\left(\gamma_{2}\right)$. When $\tau\left(\gamma_{1}\right)=$ $\tau\left(\gamma_{2}\right)$, Theorem 7 implies that $\left\{G_{\gamma_{1}}, G_{\gamma_{2}}\right\}$ is pairwise poorly convex on $[0,1]$. On the other hand, when $\tau\left(\gamma_{1}\right)<\tau\left(\gamma_{2}\right)$, with the aid of 290 we get

$$
\left(\frac{G_{\gamma_{1}}^{\prime}(t)}{G_{\gamma_{2}}^{\prime}(t)}\right)^{\prime}=\frac{\left[\sum_{i=1}^{n}\left(v_{i}-w_{i}\right)\right]^{3} \sin \left(\gamma_{2}-\gamma_{1}\right)}{\left[G_{\gamma_{2}}^{\prime}(t)\right]^{2}}<0 \quad \text { for } \tau\left(\gamma_{1}\right)<t<\tau\left(\gamma_{2}\right) \text {, }
$$

and so $G_{\gamma_{1}}^{\prime}(\cdot) / G_{\gamma_{2}}^{\prime}(\cdot)$ is decreasing in $\left(\tau\left(\gamma_{1}\right), \tau\left(\gamma_{2}\right)\right)$. Consequently, by Theo$\operatorname{rem} 9,\left\{G_{\gamma_{1}}, G_{\gamma_{2}}\right\}$ is also pairwise poorly convex on $[0,1]$ in the second case. 
Thus the family $\mathcal{G}^{*}$ is pairwise poorly convex on $[0,1]$. This implies that $\mathcal{G}$ and hence also $\mathcal{F}$ are pairwise poorly convex on $\mathbb{U}$.

EXAmple 4 . Let $\mathbb{U}=\left\{\bar{x} \in \mathbb{R}^{n}: \sum_{i=1}^{n} x_{i}^{2} \leq 1 / 2\right\}$. Consider the family $\mathcal{H}=\left\{h_{\beta}: 1 / 2 \leq \beta \leq 3 / 2\right\}$ of continuous functions $h_{\beta}$ on $\mathbb{U}$ defined by

$$
h_{\beta}(\bar{x})=-\exp \left\{-\sum_{i=1}^{n}\left(x_{i}-\beta\right)^{2}\right\} .
$$

We will show that $\mathcal{H}$ is a pairwise poorly convex family on $\mathbb{U}$.

It is easy to check that for $1 / 2<\beta<\sqrt{2}$ the function $f(t):=h_{\beta}(t, 0, \ldots, 0)$ is strictly concave and strictly convex on $[-\sqrt{2} / 2,-\sqrt{2} / 2+\beta]$ and $[-\sqrt{2} / 2+$ $\beta, \sqrt{2} / 2]$, respectively, and $f$ is strictly concave on $[-\sqrt{2} / 2, \sqrt{2} / 2]$ for $\sqrt{2} \leq$ $\beta \leq 3 / 2$. Therefore the functions $h_{\beta}$ (with $1 / 2<\beta \leq 3 / 2$ ) are not convex on $\mathbb{U}$, and Proposition 3(a) cannot be applied to show the pairwise poor convexity of $\mathcal{H}$.

Let $h_{\beta} \in \mathcal{H}$ and fix $v, w \in \partial \mathbb{U}$ with $v=\left(v_{1}, \ldots, v_{n}\right), w=\left(w_{1}, \ldots, w_{n}\right)$ and $v \prec w$. Then

$$
\sum_{i=1}^{n} v_{i}^{2}=1 / 2, \quad \sum_{i=1}^{n} w_{i}^{2}=1 / 2,
$$

and the cross function $H_{\beta}(t)=\left(h_{\beta}\right)_{v}^{w}(t)$ is the continuous function

$$
H_{\beta}(t)=-\exp \left\{-\sum_{i=1}^{n}\left[(1-t) v_{i}+t w_{i}-\beta\right]^{2}\right\}, \quad 0 \leq t \leq 1 .
$$

Obviously $\mathbb{U}$ is a compact convex subset of $\mathbb{R}^{n}$. Therefore, in view of Theorem 12 and Remark 6 , the family $\mathcal{H}$ is pairwise poorly convex if $\left\{H_{\beta}: 1 / 2 \leq\right.$ $\beta \leq 3 / 2\}$ is pairwise poorly convex on $[0,1]$.

It can be easily checked that for $t \in[0,1]$,

$$
\begin{aligned}
& H_{\beta}^{\prime}(t)=-2 \sum_{i=1}^{n}\left(w_{i}-v_{i}\right)^{2} \cdot H_{\beta}(t) \cdot\left(t-t_{\beta}\right) \\
& H_{\beta}^{\prime \prime}(t)=-2 \sum_{i=1}^{n}\left(w_{i}-v_{i}\right)^{2} \cdot H_{\beta}(t)\left[1-2 \sum_{i=1}^{n}\left(w_{i}-v_{i}\right)^{2} \cdot\left(t-t_{\beta}\right)^{2}\right],
\end{aligned}
$$

where

$$
t_{\beta}=\beta \cdot \frac{\sum_{i=1}^{n}\left(w_{i}-v_{i}\right)}{\sum_{i=1}^{n}\left(w_{i}-v_{i}\right)^{2}}-\frac{\sum_{i=1}^{n} v_{i}\left(w_{i}-v_{i}\right)}{\sum_{i=1}^{n}\left(w_{i}-v_{i}\right)^{2}} .
$$

By (31), the last equality can be simplified to

$$
t_{\beta}=\beta \cdot T+1 / 2 \quad \text { with } \quad T=\frac{\sum_{i=1}^{n}\left(w_{i}-v_{i}\right)}{1-2 \sum_{i=1}^{n} w_{i} v_{i}} .
$$


Let us define

$$
\tau_{\beta}= \begin{cases}0 & \text { if } t_{\beta}<0 \\ t_{\beta} & \text { if } 0 \leq t_{\beta} \leq 1 \\ 1 & \text { if } t_{\beta}>1\end{cases}
$$

Since $H_{\beta}(t)<0$ for $0 \leq t \leq 1$ and $\sum_{i=1}^{n}\left(w_{i}-v_{i}\right)^{2}>0$ (because $v \neq w$ ), one can easily deduce from (32) that $H_{\beta}$ is strictly decreasing in $\left[0, \tau_{\beta}\right]$ and strictly increasing in $\left[\tau_{\beta}, 1\right]$. Hence, by Theorems 6 and 11 , for $1 / 2 \leq \beta \leq 3 / 2$, $H_{\beta}$ is continuous and poorly convex on $[0,1]$, and obviously (see $(9)$ and $(10 p)$ for all $\beta$ we have

$$
\tau_{H_{\beta}}=\tau^{H_{\beta}}=\tau_{\beta} .
$$

Now fix $\beta_{1}, \beta_{2}$ in $[1 / 2,3 / 2], \beta_{1} \neq \beta_{2}$, and assume that $t_{\beta_{1}}<t_{\beta_{2}}$. We will show that $\left\{H_{\beta_{1}}, H_{\beta_{2}}\right\}$ is pairwise poorly convex on $[0,1]$.

First notice that if

$$
t_{\beta_{1}}, t_{\beta_{2}} \geq 1 \quad \text { or } \quad t_{\beta_{1}}, t_{\beta_{2}} \leq 0
$$

then $\tau_{\beta_{1}}=\tau_{\beta_{2}}=1$ or $\tau_{\beta_{1}}=\tau_{\beta_{2}}=0$, respectively, and Theorem 7 implies that $\left\{H_{\beta_{1}}, H_{\beta_{2}}\right\}$ is pairwise poorly convex on $[0,1]$. Therefore we can assume that (36) does not hold, which easily implies that

$$
t_{\beta_{1}} \leq \tau_{\beta_{1}}<\tau_{\beta_{2}} \leq t_{\beta_{2}}
$$

because $t_{\beta_{1}}<t_{\beta_{2}}$.

Now suppose that $t_{\beta_{1}}<1$ and $t_{\beta_{2}} \geq 1$. Then, in view of $(34), T<\frac{1}{2 \beta_{1}}$ and $T \geq \frac{1}{2 \beta_{2}}$. Hence $0<T<1$, because $\beta_{1}, \beta_{2} \geq 1 / 2$. Similarly we show that if $t_{\beta_{1}} \leq 0$ and $t_{\beta_{2}}>0$, then $-1<T<0$, and if $-1<t_{\beta_{1}}, t_{\beta_{2}}<1$, then $0<T<1$. Consequently, $-1<T<1$ when (36) does not hold, whence (34) and the inequality $t_{\beta_{1}}<t_{\beta_{2}}$ and $\left|\beta_{2}-\beta_{1}\right| \leq 1$ imply that

$$
0<t_{\beta_{2}}-t_{\beta_{1}}<1 \text {. }
$$

Let $J(t)=H_{\beta_{1}}^{\prime}(t) / H_{\beta_{2}}^{\prime}(t)$. With the aid of $(32)$ and $(33)$ we easily check that for $t \in[0,1]$,

$$
J^{\prime}(t)=K(t) \cdot\left(t_{\beta_{1}}-t_{\beta_{2}}\right) \cdot\left[1+2 \sum_{i=1}^{n}\left(w_{i}-v_{i}\right)^{2} \cdot\left(t-t_{\beta_{1}}\right) \cdot\left(t-t_{\beta_{2}}\right)\right],
$$

where $K(t)>0$ for all $t$.

Note that for $t \in\left(t_{\beta_{1}}, t_{\beta_{2}}\right)$, inequality (38) implies

$$
\left(t-t_{\beta_{1}}\right) \cdot\left(t-t_{\beta_{2}}\right) \geq-\left(t_{\beta_{2}}-t_{\beta_{1}}\right)^{2} / 4 \geq-1 / 4,
$$

and further

$$
\sum_{i=1}^{n}\left(w_{i}-v_{i}\right)^{2} \leq 2 \sum_{i=1}^{n}\left(w_{i}^{2}+v_{i}^{2}\right)=2
$$


because of (31). Hence, for $t \in\left(t_{\beta_{1}}, t_{\beta_{2}}\right)$ we get

$$
1+2 \sum_{i=1}^{n}\left(w_{i}-v_{i}\right)^{2} \cdot\left(t-t_{\beta_{1}}\right) \cdot\left(t-t_{\beta_{2}}\right) \geq 1+2 \cdot 2 \cdot(-1 / 4)=0 .
$$

Thus, in view of $(39)$ and $(37), J^{\prime}(t) \leq 0$ for $t \in\left(\tau_{\beta_{1}}, \tau_{\beta_{2}}\right)$, and so $H_{\beta_{1}}^{\prime} / H_{\beta_{2}}^{\prime}$ is nonincreasing in $\left(\tau_{\beta_{1}}, \tau_{\beta_{2}}\right)$. Moreover, $H_{\beta}$ is continuous and poorly convex on $[0,1]$ as shown before. Hence, in view of $(35)$, Theorem 9 shows that $\left\{H_{\beta_{1}}, H_{\beta_{2}}\right\}$ is pairwise poorly convex on $[0,1]$. Now Theorem 12 yields the pairwise poor convexity of $\mathcal{H}$.

7. Application to an optimization problem. In this section, we apply the theory developed so far to prove three theorems related to a classical optimization problem in game theory. Its solution can be interpreted as an equilibrium in various economic game models.

Throughout this section, we assume that $X$ and $Y$ are nonempty convex compact subsets of $\mathbb{R}^{k}$ and $\mathbb{R}^{l}$, respectively, $k, l \geq 1$. We denote by $P_{X}$ and $P_{Y}$ the classes of all probability distributions over $X$ and $Y$. The probability distribution with all mass concentrated at a point $t$ will be denoted by $\delta_{t}$; in some contexts $\delta_{t}$ will be identified with $t$.

Let us consider the following general problem: For two functions $K_{1}$ and $K_{2}$ defined on $X \times Y$, we look for the pair $\left(x^{*}, y^{*}\right) \in X \times Y$, called a $(N a s h)$ equilibrium, satisfying

$$
\max _{x \in X} K_{1}\left(x, y^{*}\right)=K_{1}\left(x^{*}, y^{*}\right) \text { and } \max _{y \in Y} K_{2}\left(x^{*}, y\right)=K_{2}\left(x^{*}, y^{*}\right) .
$$

The interpretation of this equilibrium concept can be the following: There are two agents I and II who choose (simultaneously and independently) elements $x \in X$ and $y \in Y$, respectively, as their strategies. By assumption, agents I and II are interested in making the values of $K_{1}(x, y)$ and $K_{2}(x, y)$, respectively, as large as possible. Therefore any equilibrium $\left(x^{*}, y^{*}\right)$ satisfying (40) describes a pair of "stable" agents' strategies, in the sense that if they agree on them, nobody is interested in changing their strategy. It is known (Glicksberg (1952)) that if $K_{1}$ and $K_{2}$ are continuous on $X \times Y$ and if $K_{1}(\cdot, y)$ and $K_{2}(x, \cdot)$ are quasi-concave for $x \in X$ and $y \in Y$, respectively, then at least one equilibrium exists. However, in general such an equilibrium may not exist. Therefore, some extension of the above model can be considered. Namely, assume that agents I and II can choose their strategies $x$ and $y$ at random from the sets $X$ and $Y$, respectively. Consequently, their strategies are probability distributions over $X$ and $Y$, respectively. We describe this extension in more detail.

Assume that the agents choose strategies $\mu \in P_{X}$ and $\nu \in P_{Y}$, respectively. We can then extend the domains of $K_{1}$ and $K_{2}$ from $X \times Y$ to $P_{X} \times P_{Y}$ 
and replace their utilities by their expected values defined by

$$
K_{i}(\mu, \nu):=\int_{X} \int_{Y} K_{i}(x, y) d \nu(y) d \mu(x), \quad i=1,2 .
$$

Now we are ready to define a natural generalization of the notion of equilibrium.

Definition 7. A pair $\left(\mu^{*}, \nu^{*}\right) \in P_{X} \times P_{Y}$ is called a generalized equilibrium if

$$
\max _{\mu \in P_{X}} K_{1}\left(\mu, \nu^{*}\right)=K_{1}\left(\mu^{*}, \nu^{*}\right) \text { and } \max _{\nu \in P_{Y}} K_{2}\left(\mu^{*}, \nu\right)=K_{2}\left(\mu^{*}, \nu^{*}\right) .
$$

REMARK 9. Glicksberg (1952) showed with the aid of Kakutani's fixed point theorem that a generalized equilibrium always exists if $K_{1}$ and $K_{2}$ are continuous on $X \times Y$. However, when they are discontinuous, there are no general methods for finding a generalized equilibrium (if it exists). Also, an agent can be interested in looking for an equilibrium with the simplest structure, which should be easier to find and implement. The three theorems below discuss such a possibility, under very weak assumptions on $K_{1}$ and $K_{2}$.

Before formulating the main results of this section note that the "optimality condition" (41) for $\left(\mu^{*}, \nu^{*}\right)$ can be simplified to

$$
\max _{x \in X} K_{1}\left(x, \nu^{*}\right)=K_{1}\left(\mu^{*}, \nu^{*}\right) \text { and } \max _{y \in Y} K_{2}\left(\mu^{*}, y\right)=K_{2}\left(\mu^{*}, \nu^{*}\right) .
$$

TheOREM 15. Assume that for any $x \in X$ the function $K_{2}(x, \cdot)$ is upper semicontinuous on $Y$, and $\left\{K_{1}(\cdot, y): y \in Y\right\}$ is a pairwise poorly concave family of upper semicontinuous functions on $X$. If there exists a generalized equilibrium $\left(\mu^{*}, \nu^{*}\right) \in P_{X} \times P_{Y}$, then for some $y_{1}, \ldots, y_{k+1} \in Y$ and $0 \leq$ $\beta_{1}, \ldots, \beta_{k+1} \leq 1$ with $\sum_{i=1}^{k+1} \beta_{i}=1$ there also exists a generalized equilibrium of the form $\left(\mu^{*}, \sum_{i=1}^{k+1} \beta_{i} \delta_{y_{i}}\right)$ such that

$$
K_{2}\left(\mu^{*}, \nu^{*}\right)=K_{2}\left(\mu^{*}, \sum_{i=1}^{k+1} \beta_{i} \delta_{y_{i}}\right) .
$$

Proof. By assumption, $\left(\mu^{*}, \nu^{*}\right)$ satisfies 42. Let $x^{0} \in X$ and suppose that $K_{1}\left(\mu^{*}, y\right)<K_{1}\left(x^{0}, y\right)$ for all $y$ in $\operatorname{supp}\left(\nu^{*}\right)$, the support of $\nu^{*}$. This implies (by integrating) that $K_{1}\left(\mu^{*}, \nu^{*}\right)<K_{1}\left(x^{0}, \nu^{*}\right)$, contradicting the first equality in (42).

Therefore, for any fixed $\varepsilon>0$,

$$
\sup _{y \in \operatorname{supp}\left(\nu^{*}\right)}\left[K_{1}\left(\mu^{*}, y\right)-K_{1}(x, y)+\varepsilon\right]>0 \quad \text { for all } x \in X .
$$

Let $\mathcal{T}=\left\{f_{\alpha}: \alpha \in \operatorname{supp}\left(\nu^{*}\right)\right\}$ where $f_{\alpha}: X \rightarrow \mathbb{R}$ is defined by $f_{\alpha}(x)=$ $K_{1}\left(\mu^{*}, \alpha\right)-K_{1}(x, \alpha)+\varepsilon$. Since, by assumption, the family $\left\{K_{1}(\cdot, \alpha): \alpha \in Y\right\}$ consists of upper semicontinuous functions and is pairwise poorly concave, 
Propositions 1(a) and 3(d) easily imply that $\mathcal{T}$ is also a pairwise poorly convex family of lower semicontinuous functions. Hence, by (44), $\mathcal{T}$ satisfies the assumptions of Theorem 14 with $\mathbb{U}=X$ and with $\alpha=y$ running over $\operatorname{supp}\left(\nu^{*}\right)$. Consequently, there are $0 \leq \beta_{1}, \ldots, \beta_{k+1} \leq 1$ and $y_{1}, \ldots, y_{k+1}$ in $\operatorname{supp}\left(\nu^{*}\right)$ such that $\sum_{i=1}^{k+1} \beta_{i} f_{y_{i}}(x)>0$ for all $x \in X$. Hence

$$
\sum_{i=1}^{k+1} \beta_{i} K_{1}\left(\mu^{*}, y_{i}\right) \geq \sum_{i=1}^{k+1} \beta_{i} K_{1}\left(x, y_{i}\right) \quad \text { for all } x \in X,
$$

or equivalently

$$
K_{1}\left(\mu^{*}, \sum_{i=1}^{k+1} \beta_{i} \delta_{y_{i}}\right) \geq K_{1}\left(x, \sum_{i=1}^{k+1} \beta_{i} \delta_{y_{i}}\right) \quad \text { for all } x \in X .
$$

Now we will show that

$$
K_{2}\left(\mu^{*}, \nu^{*}\right)=K_{2}\left(\mu^{*}, y_{i}\right) \quad \text { for } i=1, \ldots, k+1 .
$$

Firstly suppose that

$$
K_{2}\left(\mu^{*}, \nu^{*}\right)>K_{2}\left(\mu^{*}, y_{m}\right) \quad \text { for some } m, 1 \leq m \leq n+1 .
$$

Since, by assumption, for any $x \in X$ the function $K_{2}(x, \cdot)$ is upper semicontinuous on $Y$, Fatou's lemma (in the "limsup" version) easily implies that also $K_{2}\left(\mu^{*}, \cdot\right)$ is upper semicontinuous on $Y$. Hence, by Definition 2, the set $A:=\left\{y \in Y: K_{2}\left(\mu^{*}, y\right)<K_{2}\left(\mu^{*}, \nu^{*}\right)\right\}$ is (relatively) open in $Y$, and $y_{m} \in A$.

Let $B:=\operatorname{supp}\left(\nu^{*}\right)$. Since $B$ is a closed set, so is $B \backslash A$, and we have

$$
K_{2}\left(\mu^{*}, y\right) \geq K_{2}\left(\mu^{*}, \nu^{*}\right) \quad \text { for all } y \in B \backslash A,
$$

and $B \backslash A \subsetneq B$ because $y_{m} \in A$ and $y_{m} \in B$.

The equality $\nu^{*}(B \cap A)=0$ cannot hold, since then $\nu^{*}(B \backslash A)=1$, which contradicts the definition of $B$ (as a minimal closed set $M$ satisfying $\left.\nu^{*}(M)=1\right)$. Therefore $\nu^{*}(B \cap A)>0$.

Similarly, $\nu^{*}(B \cap A)=1$ cannot hold either, because it would also lead to a contradiction:

$$
K_{2}\left(\mu^{*}, \nu^{*}\right)=\int_{B \cap A} K_{2}\left(\mu^{*}, y\right) d \nu^{*}(y)<\int_{B \cap A} K_{2}\left(\mu^{*}, \nu^{*}\right) d \nu^{*}(y)=K_{2}\left(\mu^{*}, \nu^{*}\right) .
$$

Therefore $0<\nu^{*}(B \cap A)<1$.

Set $\gamma:=\nu^{*}(B \cap A)$. Then $\nu^{*}(B \backslash A)=1-\gamma$ and $0<\gamma<1$. Further, let $s \in B \backslash A$. By the definition of $A$ and $(48), K_{2}\left(\mu^{*}, s\right)>K_{2}\left(\mu^{*} y\right)$ for all $y \in B \cap A$. Now define the probability distribution $\nu^{\prime}$ in $P_{Y}$ by $\nu^{\prime}=\nu^{*}+\gamma \delta_{s}$ 
on $B \backslash A$ and $\nu^{\prime}=0$ elsewhere. Hence,

$$
\begin{aligned}
K_{2}\left(\mu^{*}, \nu^{*}\right) & =\int_{B} K_{2}\left(\mu^{*}, y\right) d \nu^{*}(y) \\
& =\int_{B \backslash A} K_{2}\left(\mu^{*}, y\right) d \nu^{*}(y)+\int_{B \cap A} K_{2}\left(\mu^{*}, y\right) d \nu^{*}(y) \\
& <\int_{B \backslash A} K_{2}\left(\mu^{*}, y\right) d \nu^{*}(y)+\gamma K_{2}\left(\mu^{*}, s\right) \\
& =\int_{B \backslash A} K_{2}\left(\mu^{*}, y\right) d\left(\nu^{*}(y)+\gamma \delta_{s}(y)\right)=K_{2}\left(\mu^{*}, \nu^{\prime}\right),
\end{aligned}
$$

so $K_{2}\left(\mu^{*}, \nu^{*}\right)<K_{2}\left(\mu^{*}, \nu^{\prime}\right)$, contradicting the second equality in 41). Thus, we have shown that (47) cannot hold. In view of the second equality in (42), this implies 46), and hence 43).

Now, it suffices to combine (45), the second equality in $(42)$ and 46$)^{\prime}$ to conclude that the pair $\left(\mu^{*}, \sum_{i=1}^{k+1} \beta_{i} \delta_{y_{i}}\right)$ satisfies 42 (with $\left.\nu^{*}=\sum_{i=1}^{k+1} \beta_{i} \delta_{y_{i}}\right)$, and so is a generalized equilibrium.

REMARK 10. Theorem 15 simplifies the problem of looking for a generalized equilibrium: it says that under the given assumptions we can restrict our search for equilibria to those whose second component is a probability distribution with support consisting of at most $k+1$ points. Moreover, on account of (43), this new equilibrium determines agent II's strategy which gives him the same "level of satisfaction".

Theorem 16. Assume that $\left\{K_{1}(\cdot, y): y \in Y\right\}$ and $\left\{K_{2}(x, \cdot): y \in Y\right\}$ are pairwise poorly concave families of upper semicontinuous functions on $X$ and $Y$, respectively. If there exists a generalized equilibrium in $P_{X} \times P_{Y}$, then there also exists a generalized equilibrium $\left(\mu^{0}, \nu^{0}\right)$ of the form

$$
\left(\mu^{0}, \nu^{0}\right)=\left(\sum_{i=1}^{l+1} \alpha_{i} \delta_{x_{i}}, \sum_{i=1}^{k+1} \beta_{i} \delta_{y_{i}}\right),
$$

where $x_{1}, \ldots, x_{l+1} \in X, y_{1}, \ldots, y_{k+1} \in Y$ and $0 \leq \alpha_{1}, \ldots, \alpha_{l+1}, \beta_{1}, \ldots, \beta_{k+1}$ $\leq 1$ with $\sum_{i=1}^{l+1} \alpha_{i}=1$ and $\sum_{i=1}^{k+1} \beta_{i}=1$.

Proof. Let $\left(\mu^{*}, \nu^{*}\right)$ be a generalized equilibrium in $P_{X} \times P_{Y}$. Since the assumptions of Theorem 15 are satisfied, there is a generalized equilibrium of the form $\left(\mu^{*}, \sum_{i=1}^{k+1} \beta_{i} \delta_{y_{i}}\right)$. Now, starting with this new equilibrium and applying Theorem 15 again (after changing the order of variables and functions), we get the existence of a generalized equilibrium as described in the theorem.

REMARK 11. Theorem 16 simplifies the problem of looking for a generalized equilibrium in the case when $X, Y \subset \mathbb{R}$ : we can restrict our search 
for equilibria to pairs of probability distributions with supports consisting of at most two points. Then our optimization problem can be reduced to a very simple one. Namely, taking into account $(42)$ and the fact that $X$ and $Y$ are intervals $[a, b]$ and $[c, d]$, respectively, the problem of finding a generalized equilibrium can be reduced to the following: Find six numbers $\alpha, \beta, x_{1}^{*}, x_{2}^{*}, y_{1}^{*}, y_{2}^{*}$ with $0 \leq \alpha, \beta \leq 1, a \leq x_{1}^{*}, x_{2}^{*} \leq b$ and $c \leq y_{1}^{*}, y_{2}^{*} \leq d$ satisfying

$$
\begin{aligned}
\max _{a \leq x \leq b} & {\left[\beta K_{1}\left(x, y_{1}^{*}\right)+(1-\beta) K_{1}\left(x, y_{2}^{*}\right)\right]=\alpha \beta K_{1}\left(x_{1}^{*}, y_{1}^{*}\right) } \\
& +\alpha(1-\beta) K_{1}\left(x_{1}^{*}, y_{2}^{*}\right)+(1-\alpha) \beta K_{1}\left(x_{2}^{*}, y_{1}^{*}\right)+(1-\alpha)(1-\beta) K_{1}\left(x_{2}^{*}, y_{2}^{*}\right)
\end{aligned}
$$

and

$$
\begin{aligned}
\max _{c \leq y \leq d} & {\left[\alpha K_{2}\left(x_{1}^{*}, y\right)+(1-\alpha) K_{2}\left(x_{2}^{*}, y\right)\right]=\alpha \beta K_{2}\left(x_{1}^{*}, y_{1}^{*}\right) } \\
& +\alpha(1-\beta) K_{2}\left(x_{1}^{*}, y_{2}^{*}\right)+(1-\alpha) \beta K_{2}\left(x_{2}^{*}, y_{1}^{*}\right)+(1-\alpha)(1-\beta) K_{2}\left(x_{2}^{*}, y_{2}^{*}\right) .
\end{aligned}
$$

Then the pair $\left[\alpha \delta_{x_{1}^{*}}+(1-\alpha) \delta_{x_{2}^{*}}, \beta \delta_{y_{1}^{*}}+(1-\beta) \delta_{y_{2}^{*}}\right]$ is an equilibrium. Obviously, this task is much easier than looking for a generalized equilibrium $\left(\mu^{*}, \nu^{*}\right)$ satisfying (41) over the spaces $P_{X}$ and $P_{Y}$ of probability distributions.

We end this section with a theorem on a simplified optimization problem often discussed in game theory, where a generalized equilibrium $\left(\mu^{*}, \nu^{*}\right) \in$ $P_{X} \times P_{Y}$ is searched when $K_{2}(x, y)=-K_{1}(x, y)$ for all $(x, y) \in X \times Y$. Then, after taking $K_{1} \equiv K$, the optimality condition 42 can be equivalently rewritten in the form

$$
\max _{x \in X} K\left(x, \nu^{*}\right)=K\left(\mu^{*}, \nu^{*}\right)=\min _{y \in Y} K\left(\mu^{*}, y\right),
$$

where $K$ is a fixed function on $X \times Y$. It turns out that under this new optimization condition we have the following stronger existence theorem.

TheOREM 17. Assume that for any $x \in X$ the function $K(x, \cdot)$ is lower semicontinuous on $Y$, and $\{K(\cdot, y): y \in Y\}$ is a pairwise poorly concave family of upper semicontinuous functions on $X$. Then there exists a qeneralized equilibrium of the form $\left(\mu^{*}, \nu^{*}\right)=\left(x^{*}, \sum_{i=1}^{k+1} \beta_{i} \delta_{y_{i}^{*}}\right)$ satisfying 49 with some $x^{*} \in X, y_{1}^{*}, \ldots, y_{k+1}^{*} \in Y$ and with coefficients $0 \leq \beta_{1}, \ldots, \beta_{k+1} \leq 1$ satisfying $\sum_{i=1}^{k+1} \beta_{i}=1$.

Proof. Let us extend $K$ to $X \times P_{Y}$ by

$$
K(x, \nu)=\int_{Y} K(x, y) d \nu(y) \quad \text { for }(x, \nu) \in X \times P_{Y} .
$$

Since for every $x \in X, K(x, \cdot)$ is lower semicontinuous on the compact set $Y$, it is also lower semicontinuous on $P_{Y}$. Moreover, obviously $K(x, \cdot)$ is a convex function on $P_{Y}$. 
Therefore the assumptions of Proposition 5 are satisfied with $Y \equiv P_{Y}$ and $f=K$, and consequently

$$
\sup _{x \in X} \inf _{\nu \in P_{Y}} K(x, \nu)=\inf _{\nu \in P_{Y}} \sup _{x \in X} K(x, \nu) .
$$

Since both $X$ and $P_{Y}$ are compact, we easily conclude (using Proposition 11(d, e)) that (51) can be equivalently rewritten as

$$
\max _{x \in X} \min _{\nu \in P_{Y}} K(x, \nu)=\min _{\nu \in P_{Y}} \max _{x \in X} K(x, \nu) .
$$

Thus, there is a generalized equilibrium $\left(x^{*}, \nu^{*}\right) \in X \times P_{Y}$ such that

$$
\min _{\nu \in P_{Y}} K\left(x^{*}, \nu\right)=K\left(x^{*}, \nu^{*}\right)=\max _{x \in X} K\left(x, \nu^{*}\right) .
$$

Inequalities (42) hold for $K_{1}=K$ and $K_{2}=-K$, so we can apply Theorem 15 with $\left(\mu^{*}, \nu^{*}\right)=\left(x^{*}, \nu^{*}\right)$ to get the existence of a generalized equilibrium of the form $\left(x^{*}, \sum_{i=1}^{k+1} \beta_{i} \delta_{y_{i}^{*}}\right)$.

8. Concluding remarks. In this paper we develop a theory of poor convexity of real functions on compact convex subsets of $\mathbb{R}^{n}$. This property is intermediate between the classical convexity and quasi-convexity. A full characterization of poorly convex functions and their properties are given. This is then used to get the basic result of the paper, Theorem 14, which is a generalization (from convex continuous to poorly convex lower semicontinuous functions) of the well-known result due to Bohnenblust, Karlin and Shapley, described in Remark 7 of Section 5. Finally, an application of the theory of poorly convex functions to some optimization problems is given.

Theorem 3 says that every continuous quasi-convex function on an interval $[a, b] \subset \mathbb{R}$ can be approximated by a sequence of poorly convex continuous functions in the topology of uniform convergence. An open question is if this can be generalized to functions on a convex compact subset $\mathbb{U} \subset \mathbb{R}^{n}$ with $n>1$.

Acknowledgements. The author thanks an anonymous referee for some essential remarks and suggestions.

This research was supported by GRANT 342667/I-18 of Wrocław University of Technology.

\section{References}

K. J. Arrow and A. C. Enthoven (1961), Quasiconcave programming, Econometrica 29, 779-800.

H. F. Bohnenblust, S. Karlin, and L. S. Shapley (1950), Games with Continuous Convex Payoff, Ann. of Math. Stud. 24, Princeton Univ. Press, 181-192. 
J. Eckhoff (1993), Helly, Radon, and Carathéory type theorems, in: Handbook of Convex Geometry, Vol. A, North-Holland, Amsterdam, 389-448.

W. Fenchel (1953), Convex Cones, Sets and Functions, Lecture Notes, Princeton Univ.

B. de Finetti (1949), Sulle stratificazioni convesse, Ann. Mat. Pura Appl. 30, 173-183.

[. Glicksberg (1952), A further generalization of the Kakutani fixed points theorem with application to Nash equilibrium points, Proc. Amer. Math. Soc. 38, 170-174.

A. Guerraggio and E. Molho (2004), The origin of quasi-concavity: a development between mathematics and economics, Historia Math. 31, 62-75.

M. Hanson (1981), On sufficiency of the Kuhn-Tucker conditions, J. Math. Anal. Appl. $80,545-550$.

5. M. N. Islam and D. B. Craven (2005), Some extensions of nonconvex economic modeling invexity, quasimax and new stability conditions, J. Optim. Theory Appl. 125, 315-330.

5. Karamardian (1967), Strictly quasi convex (concave) functions and duality in mathematical programming, J. Math. Anal. Appl. 20, 344-358.

J. L. Mangasarian (1965), Pseudo-convex functions, SIAM J. Control 3, 281-290.

J. Matkowski (2008), Generalized convex functions and a solution of a problem of Zs. Páles, Publ. Math. Debrecen 73, 421-460.

S. K. Mishra, S. Y. Wang and K. Lai (2009), Generalized Convexity and Vector Optimization, Springer, Berlin.

T. Radzik (2014), Poor convexity and Nash equilibria in games, Int. J. Game Theory 43, 169-192.

M. Sion (1958), On general minimax theorems, Pacific J. Math. 8, 171-176.

Tadeusz Radzik

Faculty of Mathematics

Wrocław University of Technology

Wybrzeże Wyspiańskiego 27

50-370 Wrocław, Poland

E-mail: tadeusz.radzik@pwr.edu.pl 
